\title{
UMA ABORDAGEM FUZZY PARA A AVALIAÇÃO TÉCNICO-ECONÔMICA DE REDES DE ACESSO
}

\begin{abstract}
Marcos A. DeSousa*
mas@ucg.br

Joana T. Machado $\ddagger$

joana@densis.fee.unicamp.br

\section{RESUMO}

O surgimento de novas tecnologias e serviços vem impondo mudanças substanciais ao tradicional sistema de telecomunicações. Múltiplas possibilidades de evolução do sistema fazem da etapa de planejamento um procedimento não só desejável como necessário, principalmente num ambiente de competitividade. A utilização de metodologias abrangentes e flexíveis que possam auxiliar no processo de decisão, fundadas em modelos de otimização, parece um caminho inevitável. Este artigo propõe um modelo de programação linear inteiro misto para ajudar no planejamento estratégico de sistemas de telecomunicações, e em particular da rede de acesso. Os principais componentes de custo e receita são identificados e o modelo é desenvolvido para determinar a configuração da rede (serviços, tecnologias, etc) que maximize a receita esperada pelo operador do sistema. $\mathrm{O}$ conceito de números fuzzy é adotado para avaliar o risco técnico-econômico em situações de imprecisão nos dados de demanda. Resultados de experimentos computacionais são apresentados e discutidos.
\end{abstract}

*Departamento de Engenharia, Universidade Católica de Goiás (UCG)
Av. Universitária, 1440, Área III, Bloco G, Sala 108, CEP 74605-010, Goiânia-GO, Brasil

${ }^{\dagger}$ Departamento de Ciências de Computação e Estatística, Universidade Estadual Paulista (IBILCE / UNESP)

\author{
Carlos M. F. Carlson ${ }^{\dagger}$ \\ carlson@dcce.ibilce.unesp.br \\ Raul V. Ribeiro ${ }^{\ddagger}$ \\ raul@densis.fee.unicamp.br
}

${ }^{\ddagger}$ Faculdade de Engenharia Elétrica e de Computação (DENSIS/FEEC), Universidade Estadual de Campinas (UNICAMP)

\footnotetext{
Artigo submetido em 05/12/2003

1a. Revisão em 18/10/2004

2a. Revisão em 19/04/2006

Aceito sob recomendação do Editor Associado

Prof. Cairo Lucio Nascimento Jr
}

PALAVRAS-CHAVE: Sistemas de telecomunicações, modelagem matemática, fuzzy sets.

\section{ABSTRACT}

The rising of new technologies and services imposes significant changes to the traditional telecommunications system. The diversity of system evolution settings makes the planning stage an increasingly desirable procedure, mainly in a competitive environment. The use of comprehensive and flexible methodologies that can support the decision process, based in optimization mathematical models, seems to be indispensable. This paper proposes a mixed-integer linear programming model devoted to help the strategic planning of the telecommunication systems, and in the special of the access network. Major components of costs and revenues are identified. The model intends to determine the network configuration (services, technologies, etc.) which maximizes the expected revenue. In order to perform some techno-economical risk analyses in situations with lack of precision in the demand data, fuzzy sets concepts are adopted. Results of computational experiments are presented and discussed.

KEYWORDS: Telecommunication systems, Mathematical models, fuzzy sets. 


\section{INTRODUÇÃO}

O desenvolvimento assistido pelas indústrias de microeletrônica e informática permite oferecer diversas soluções tecnológicas, as quais devem ser consideradas no gerenciamento de informação. Tecnologias que até recentemente tinham aplicação apenas em segmentos específicos da rede, como por exemplo um backbone de entroncamento entre centrais telefônicas, já se apresentam economicamente viáveis para o oferecimento de serviços multimídia ao usuário final, com melhor qualidade de transmissão e com preços mais acessíveis.

Tecnologias baseadas em fibras ópticas tendem a ser dominantes para situações que exigem grande volume de informação e alta confiabilidade da rede. Entretanto, soluções que permitem aproveitar os cabos de cobre para transmitir informações em altas velocidades, garantindo uma "sobrevida"a uma eventual rede já existente, precisam ser analisadas. Outra opção, não menos importante, é usar também enlaces via rádio.

Estas mudanças levam as empresas operadoras a assumirem novas posturas em todas as suas áreas de atuação, inclusive no que diz respeito ao planejamento da expansão do sistema, principalmente no segmento de acesso do usuário, onde a diversidade tecnológica e de serviços tende a ser mais acentuada (Toledo, 2001; Yacoub, 2001; Ims et alii 1998).

O cenário mercadológico atual, no qual a exploração dos serviços está a cargo de empresas de capital privado, sugere mudanças nas metodologias correntes de planejamento. A competição faz com que o dimensionamento dos sistemas contemple fatores técnicos e econômicos que vão além da simples tarefa de planejar a rede objetivando o custo mínimo, seja de implantação, aluguel, manutenção e/ou operação. Implantar soluções que signifiquem garantia de participação no mercado e receitas compensadoras é uma tendência necessária e inevitável.

Isto traz novas preocupações para a etapa de planejamento. Por um lado, é possível haver seletividade no atendimento da demanda, o que significa dizer que as demandas potencialmente mais lucrativas serão prioritárias. Por outro lado, existe a variedade de serviços a oferecer, cada qual gerando receita diferenciada e, eventualmente, exigindo equipamentos, topologias e meios de transmissão específicos. A limitação orçamentária, naturalmente, é outro fator a ser previsto, pois nem sempre é possível implantar todos os sistemas necessários ao atendimento pleno da demanda.

Algumas abordagens para o planejamento técnicoeconômico de sistemas de telecomunicações podem ser encontradas na literatura. Na linha de pesquisa orientada à minimização de custo, contemplando várias tecnologias de acesso, pode-se citar os trabalhos de Olsen et alii (1996) e Balakrishnan et alii (1991). Alguns modelos matemáticos de aplicação mais específica também foram desenvolvidos em estudos mais recentes. Análises de custo para a infraestrutura de sistemas de acesso sem fio podem ser vistas em DeSousa et alii (2001) e Ufongene (1999), enquanto Carlson e Authié (2001) estudam o dimensionamento de sistemas LMDS (Local Multipoint Distribution Service). Uma avaliação multi-período para o problema de planejamento de sistemas de acesso celular é assunto do trabalho desenvolvido por Kubat e Smith (2001). Em Moura e Garcia (2000) é proposta uma modelagem para a alocação e dimensionamento de equipamentos de uma rede multicamadas, que utiliza a tecnologia SDH (Synchronous Digital Hierarchy) como meio de transporte para a tecnologia ATM (Asynchronous Transfer Mode).

O planejamento técnico-econômico orientado à maximização de receita também tem sido objeto de diversos estudos. Uma análise dos aspectos econômicos no planejamento de sistemas de acesso sem fio, embora sem focalizar nenhuma tecnologia específica, pode ser encontrada em Gavish e Sridhar (1995). Alguns aspectos sobre a alocação de recursos em um sistema CDMA (Code Division Multiple Access) são endereçados em Zhou et alii (2003). Um estudo sobre a escolha de tecnologia e alocação de equipamentos para redes de acesso celulares, utilizando algoritmos genéticos no processo de resolução dos modelos matemáticos, pode ser vista em Machado et alii (2003). Outros modelos de otimização aplicados ao dimensionamento de equipamentos e avaliação técnico-econômica de sistemas de acesso multi-serviço são propostos em Desousa et alii (2003, 2001a, 1999).

Entretanto, uma característica bastante comum entre estas metodologias de planejamento é o fato de que o dimensionamento da rede depende fortemente da precisão com que os dados de entrada são apresentados aos modelos. Assim, os dados, como por exemplo a penetração (aceitação) de um determinado serviço, são elementos fundamentais que precisam ser fornecidos pelo planejador. Porém, estas informações “precisas” nem sempre estão disponíveis durante a etapa de planejamento, o que é natural num contexto de rápido desenvolvimento tecnológico e surgimento de novos serviços. $\mathrm{O}$ resultado do planejamento, portanto, pode ser sensível à variação destes dados de entrada. A ferramenta computacional desenvolvida para este fim também precisa estar preparada para absorver estas "imprecisões" existentes nos dados do planejamento.

Como primeira conseqüência dos dados imprecisos alguns parâmetros dos modelos, baseados nesses dados, não são mais fixos. A maneira de resolver o problema matemático, não mais "exato", pode mudar consideravelmente. A idéia natural é tentar manter o problema passível de solução, incor- 
porando ao mesmo tempo aspectos da imprecisão no sentido de flexibilizar o modelo e torná-lo mais fiel ao ambiente que pretende retratar. As técnicas mais usadas são a programação estocástica e a aplicação de conceitos de conjuntos nebulosos. Outra opção é utilizar intervalos para a representação de valores numéricos. Vários trabalhos sobre o assunto são relatados no survey organizado por Sahinidis (2004).

Neste trabalho, propõe-se um modelo matemático para ajudar no planejamento estratégico de sistemas de acesso, em particular, para a rede de alimentação do sistema de acesso fixo e/ou para a infra-estrutura do sistema de acesso móvel celular. Ele reflete o ambiente de competição entre as tecnologias e os serviços. O dimensionamento é feito com o objetivo de se obter a maior receita possível dos serviços oferecidos, respeitando as demandas previstas, topologias de rede, capacidades e custos das tecnologias candidatas, bem como as condições orçamentárias do investidor. Facilidades existentes, como dutos e cabos, também são incluídos como participantes do processo de decisão. Com o objetivo de obter um modelo mais robusto, capaz de avaliar o risco técnicoeconômico de se investir em diferentes cenários de penetração dos serviços, o conceito de número fuzzy também é agregado à metodologia (Desousa et alii 2003a, 2001b, 2000).

\subsection{Sistema de Acesso}

A rede de acesso representa a "porta de entrada"de cada usuário (denominado assinante) do sistema de telecomunicações. Tradicionalmente, no acesso fixo a conexão é feita através de cabos de cobre que conectam o assinante a um ponto de concentração, o nó de acesso. Esse segmento da rede recebe o nome de rede de distribuição. A partir do nó de acesso, os assinantes são ligados à Estação Telefônica (ET) por meio de enlaces de maior capacidade e/ou são concentrados de maneira a compartilhar o mesmo meio de transmissão. Esse segmento recebe o nome de rede de alimentação. A rede de distribuição e a rede de alimentação constituem o sistema de acesso fixo (figura 1).

No sistema de acesso móvel celular (figura 2), o usuário (Mobile Station - MS) é conectado ao sistema através da interface aérea localizada na Estação Radio Base - ERB (ou Base Transceiver Station - BTS). Atualmente, as tecnologias mais adotadas neste segmento da rede são a Global System for Mobile communications (GSM) e a Code Division Multiple Acces (CDMA) (Yacoub, 2001). O planejamento da infraestrutura do sistema de acesso celular, ou seja a interconexão entre BTSs e central de comutação (ou conjunto Base Station Controller / Mobile Switching Center - BSC/MSC), pode ser feito de forma análoga ao proposto para a rede de alimentação do sistema de acesso fixo.

Em ambos os sistemas de acesso (fixo ou móvel), se dois as-

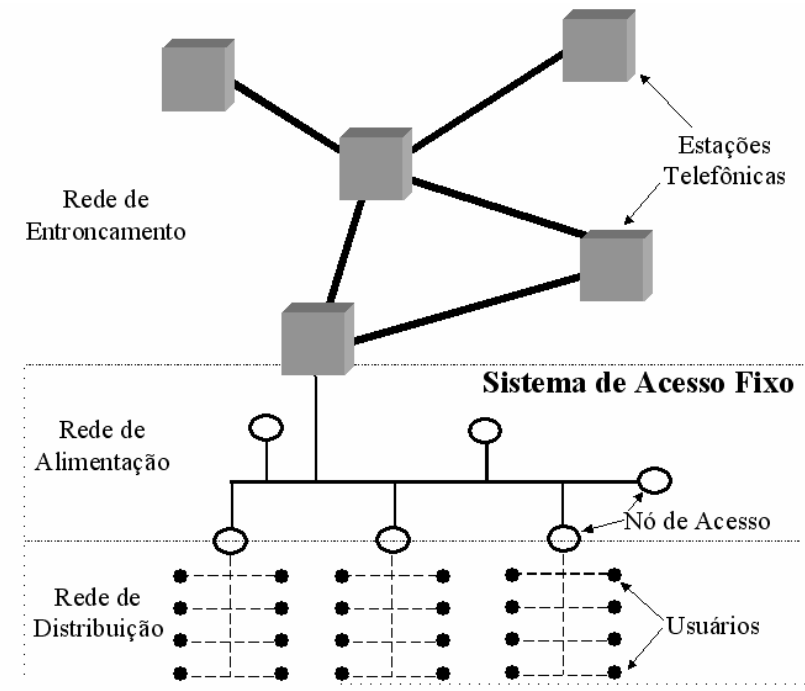

Figura 1: Componentes do sistema de acesso fixo.

sinantes não pertencem à área coberta por uma mesma central de comutação (CC), a ligação entre eles envolverá duas ou mais $\mathrm{CC}$, interligadas por uma rede de entroncamento (ou backbone). A modelagem proposta neste trabalho se aplica aos dois tipos de sistemas.

\subsection{Serviços}

Novas tecnologias de acesso, comutação e transmissão permitiram o suporte para vários tipos de serviço: voz, dados em baixa e alta velocidade, vídeo-fone, vídeo-conferência, teleeducação, etc. Seus principais atributos a serem considerados durante a etapa de planejamento do sistema de acesso são: taxa (velocidade) de transmissão, tipo de comutação (por circuito ou por pacotes), taxa de penetração (aceitação) na área em estudo, exigência (ou não) de mobilidade e a sua receita unitária (mensal ou anual).

Um dos grandes desafios das empresas de telecomunicações é definir o cardápio de serviços que elas devem oferecer. Em função destas indefinições, as metodologias de planejamento procuram organizá-los em grupos ou classes. Um critério bastante adotado é o da taxa de transmissão requerida (Ims et alii, 1997), classificando os serviços conforme a tabela 1.

Uma consequiência imediata deste agrupamento refere-se ao próprio atendimento dos serviços. É natural admitir que os serviços RDSI, FLR e FLC, que apresentam taxa de transmissão superior a $64 \mathrm{Kbps}$, incorporem também o canal de VOZ no ato de sua disponibilização. Vale salientar que esse elenco de serviços indicado não é único. No modelo proposto, ele pode ser modificado sem influenciar o seu desempenho. 


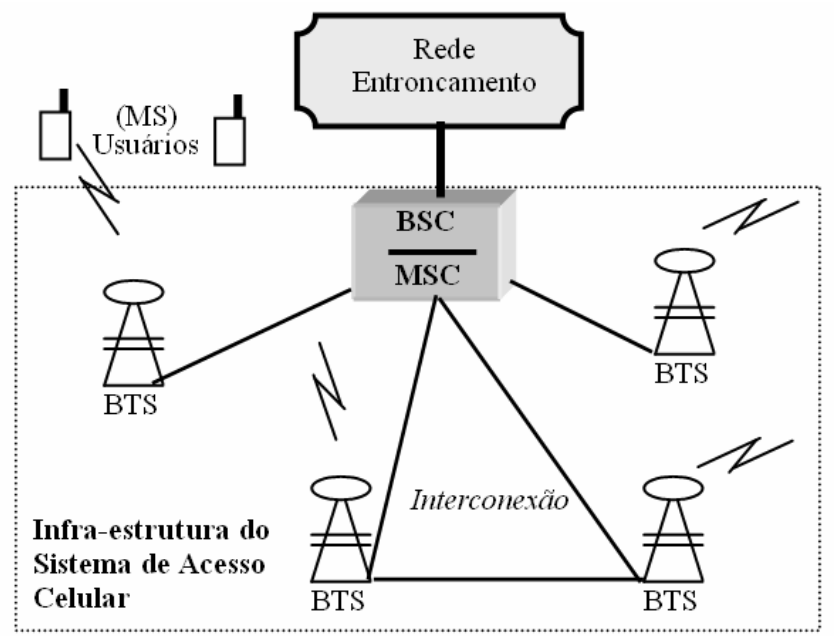

Figura 2: Componentes do sistema de acesso celular.

\begin{tabular}{|c|c|c|c|}
\hline Serviço & Categoria & Taxa Transmissão & Perfil \\
\hline $\mathrm{VOZ}^{a}$ & $\mathrm{FE}^{1}$ & $64 \mathrm{Kbps}^{3}$ & $\mathrm{SS}^{5}$ \\
\hline $\mathrm{RDSI}^{a}$ & $\mathrm{FE}$ & $144 \mathrm{Kbps}$ & $\mathrm{SS}$ \\
\hline $\mathrm{FLR}^{b}$ & $\mathrm{FL}^{2}$ & 2 a $8 \mathrm{Mbps}^{4}$ & $\mathrm{AS}^{6}$ \\
\hline $\mathrm{FLC}^{c}$ & $\mathrm{FL}$ & 2 a $8 \mathrm{Mbps}$ & $\mathrm{SS}$ \\
\hline
\end{tabular}

${ }^{a}$ Rede Digital de Serviços Integrados;

${ }^{b}$ Faixa-Larga Residencial; ${ }^{c}$ Faixa-Larga Comercial;

${ }^{1}$ Faixa Estreita; ${ }^{2}$ Faixa-Larga; ${ }^{3}$ Kilobits por segundo;

${ }^{4}$ Megabits por segundo; ${ }^{5}$ Simétrica; ${ }^{6}$ Assimétrica;

Tabela 1: Classificação dos serviços.

\subsection{Diversidade Tecnológica}

Os serviços a serem oferecidos podem, eventualmente, exigir equipamentos, topologias e meios de transmissão especificos. Para realizar a evolução do sistema, para o segmento Nó de Acesso - CC, as empresas operadoras possuem várias opções, podendo utilizar diferentes soluções tecnológicas:

Modems xDSL (Digital Subscriber Line ): é a solução ponto-a-ponto que provoca menores mudanças no sistema convencional de telefonia. O meio de transmissão é a rede metálica (par trançado). A grande mudança fica por conta dos modems de alta velocidade xDSL, os quais são colocados nas duas extremidades da rede (CC e usuário) (Toledo, 2001; Humphrey e Freeman, 1997);

Enlaces Via Rádio: é uma solução frequentemente utilizada para a interconexão de BTSs. Apresenta as seguintes capacidades: 1, 2, 4 e 8 canais E1 (taxa de transmissão = 2,048 Mbps) (Ericsson 2005; Siemens, 2005);
Modems Ópticos: é uma solução ponto-a-ponto com as mesmas opções de velocidade dos enlaces via rádio (1, 2, 4 e 8 canais E1) (Asga, 2005);

HFC (Hybrid Fiber/Coax): é uma evolução natural das redes de TV a Cabo. A adoção de uma rede mista, composta por um trecho em fibra óptica e um trecho em cabo coaxial, possibilita uma maior interatividade ao sistema (Martinelli e Carneiro, 1996);

FITL (Fiber-in-the-Loop): a viabilidade de se utilizar a fibra óptica no sistema de acesso surge com a perspectiva de demanda cada vez maior por serviços faixa-larga. Primeiramente os cabos ópticos devem ser instalados em uma topologia intermediária, permitindo o compartilhamento do sistema por vários usuários. A longo prazo pode-se vislumbrar a fibra chegando as dependências de cada usuário em particular. Dentre as tecnologias que podem usar a fibra óptica como meio de transmissão pode-se destacar a SDH e/ou a ATM (Sexton e Reid, 1997).

\section{DESCRIÇÃO DO MODELO EXATO}

Quando se pensa em planejamento orientado à receita, algumas considerações são necessárias.

\subsection{Aspectos Econômicos}

Inicialmente, é preciso lembrar que a diferentes serviços associam-se distintos valores de tarifação, a qual pode ser baseada em um valor fixo (mensal, por exemplo, como uma assinatura) ou então depender do tempo efetivo de utilização do serviço. Às vezes, uma combinação das duas formas é usada.

Por outro lado, a receita pode ser distribuída ao longo do período de tempo analisado. Neste caso, comparações com o custo fazem mais sentido quando colocadas em uma mesma unidade de medida, como por exemplo, o "valor presente" ou algum tipo de "fluxo de caixa" (Hummel e Taschner, 1992).

Um problema que envolve maximização de receita tem portanto diferentes abordagens:

\section{Maximização de receita respeitando um orçamento:} neste caso é assumido que existe um valor de receita unitária mensal (ou anual) para cada um dos serviços. A receita precisa ser maximizada, mas a pressão orçamentária pode não permitir que sejam implantados equipamentos e rede suficientes para todos. Trata-se de atender seletivamente a demanda;

Maximização da receita a custo mínimo: a receita e o custo são objetivos distintos e conflitantes. A aborda- 
gem multi-objetivo parece ser a mais adequada nesta situação;

Maximização de lucro: neste caso é preciso calcular a diferença entre receita e custo, a qual define o lucro. Assim, faz-se necessário compartilhar as respectivas unidades de medida. Como uma possibilidade, pode-se trazer o custo para seu valor presente, usando métodos de matemática financeira. A receita também precisa ser calculada na mesma base.

Neste trabalho é adotado o critério de "Maximização de Receita Respeitando um Limitante de Orçamento". No modelo, a receita unitária representa a parcela fixa da tarifa cobrada pelo oferecimento do serviço.

Para cada enlace instalado na rede, para atendimento da demanda, é possível associar um custo fixo e um custo variável. Tal flexibilidade permite a aplicação do modelo para dois padrões de cenários:

Implantação de rede: são examinados custos de implantação de uma nova rede ou da expansão de uma rede existente. Neste caso são considerados os custos de equipamentos alocados (custos fixos) e custos de cabos e dutos utilizados (custos variáveis com a distância).

Aluguel de Rede: os enlaces ponto-a-ponto são alugados pelas empresas operadoras utilizando duas componentes de custo. A primeira é uma parte fixa, com valor proporcional à capacidade do canal oferecido (geralmente múltiplos de canal E1) e a segunda é uma parte variável com a distância do enlace.

Através de um pré-processamento dos dados de custo, é possível construir cenários comparando a opção de aluguel ou implantação da rede. Para isto é necessário utilizar os conceitos básicos de engenharia econômica e converter o gasto com o aluguel para o valor presente, ou converter o custo de implantação, operação, administração e manutenção em valor uniforme líquido.

\subsection{Representação Gráfica do Sistema de Acesso}

A modelagem proposta pode ser aplicada tanto para o sistema de acesso fixo quanto para a infra-estrutura do sistema de acesso celular. Em função das previsões de demanda para os serviços, assume-se que a evolução tecnológica acontece para um único estágio de planejamento. Os assinantes podem ligar-se a apenas uma Central de Comutação (CC).

Esse comportamento da demanda e a disposição física da rede sugerem o uso de grafos (Bazaraa et alii, 1990) para a

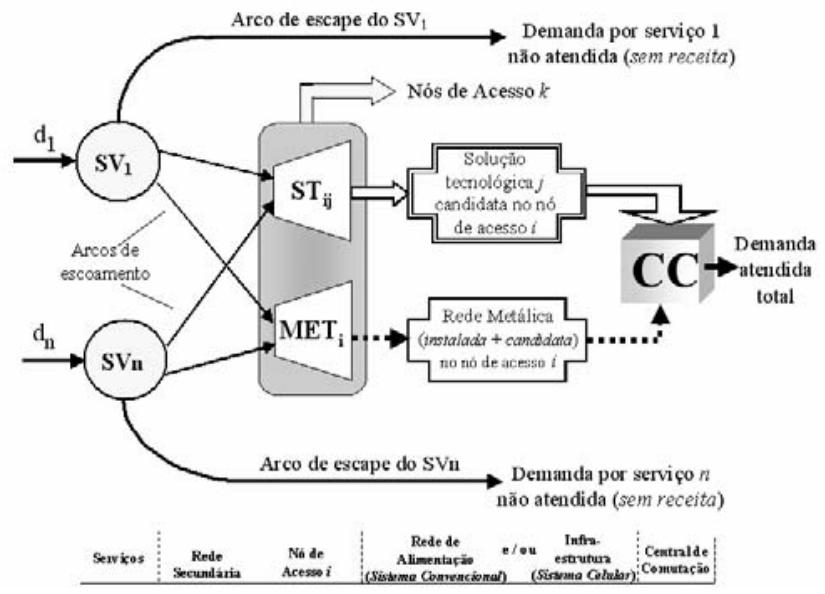

Figura 3: Representação gráfica do sistema de acesso.

sua representação. Para o planejamento em que se considera a presença de uma rede metálica instalada, esta representação para cada Nó de Acesso i pode ser conforme a figura 3. A modelagem é flexível o suficiente para permitir ao planejador escolher os seus candidatos. O nó de acesso $i$ pode ser conectado diretamente à $\mathrm{CC}$ ou apresentar rotas alternativas através dos nós de acesso $k$. Nesta representação são considerados os seguintes elementos:

Nós Artificiais de Serviço: assume-se que as demandas por cada serviço $\left(d_{1}-d_{n}\right)$ já são conhecidas. Para cada serviço existe um nó associado $\left(S V_{1}-S V_{n}\right)$, que é ligado a todos os Nós de Acesso i através de Arcos de Escoamento das demandas por esse serviço. Existe a necessidade de que as demandas apresentadas aos Nós Artificiais de Serviço tenham a mesma unidade de medida, por exemplo: número de assinantes.

Nós de Acesso: representam pontos intermediários entre o usuário do sistema e a CC. O Estágio Remoto (ER) no sistema de acesso fixo e a BTS no sistema de acesso celular são alguns exemplos. Eles funcionam como pontos de concentração, reunindo a demanda proveniente dos Nós Artificiais de Serviço. Cada Nó de Acesso i pode ser dividido em dois, um representando o acesso através da Rede Metálica $\left(\right.$ Met $\left._{i}\right)$ e outro o acesso através da Solução Tecnológica Candidata j $\left(S T_{i j}\right)$. Assim, é possível "enxergar"a rede metálica instalada, possibilitando também a sua expansão. No grafo, o Nó de Acesso $i$ também pode ser conectado a outros nós de acesso $k$. Isto configura a possibilidade de atendimento da demanda através de rotas alternativas.

Nó de Comutação: este nó está fisicamente associado à central de comutação do sistema (ET, no sistema de acesso fixo, e BSC/MSC, no sistema de acesso celular). 
Toda a demanda efetivamente atendida pelo sistema é encaminhada a esse nó.

Demanda Atendida Total: representa a demanda efetivamente atendida pelo sistema. Devido ao limite de orçamento, ela pode ser inferior à soma das demandas previstas nos Nós Artificiais de Serviço.

Arcos de Escoamento: representam os arcos que ligam todos os Nós Artificiais de Serviço $\left(S V_{1}-S V_{n}\right)$ a todos os Nós de Acesso i $\left(\mathrm{Met}_{i}+S T_{i j}\right)$. O fluxo de demanda escoado por estes arcos é utilizado tanto para contabilizar a receita gerada por cada um dos serviços oferecidos, quanto para controlar (se necessário) os gastos com a implantação da Rede Secundária. A maximização do fluxo nestes arcos para todos os serviços é o principal objetivo do modelo de otimização. O controle no atendimento dos serviços exige que os Arcos de Escoamento sejam capacitados.

Arcos de Escape: devido a um limite de orçamento, algumas demandas de certos serviços podem não ser atendidas. Por isso, Arcos de Escape são previstos para contabilizar esta demanda não atendida e garantir o balanço de fluxo nos Nós Artificiais de Serviço. A demanda escoada por estes arcos não é revertida em receita.

Rede Metálica: este segmento do grafo é utilizado para representar tanto a rede metálica já Instalada quanto a Candidata. A Instalada garante o reconhecimento da infra-estrutura disponível no sistema, tais como cabos e dutos; enquanto a Candidata representa os componentes necessários para a expansão em rede metálica. A fim de suportar também os serviços faixa-larga, este dimensionamento pode ser feito utilizando-se os modems da tecnologia XDSL.

Solução Tecnológica Candidata: representa as diversas tecnologias possíveis de serem utilizadas no planejamento do sistema, exceto a solução xDSL (candidata em conjunto com a rede metálica). Na aplicação do modelo haverá a necessidade de se adaptá-lo às características próprias de cada tecnologia. Esta adaptação pode significar a utilização de nós artificiais e/ou variáveis de decisão específicas para a alocação e dimensionamento de equipamentos.

Rede Secundária: permite o cálculo do custo associado aos equipamentos a serem disponibilizados nas instalações do usuário. Seu dimensionamento é realizado em função da demanda individual de cada serviço e da solução tecnológica candidata, o que em certas tecnologias exige considerações quanto ao dimensionamento dos equipamentos a serem alocados no Nó de Acesso. No sistema de acesso fixo, a rede secundária física (cabos) pode ser assumida como existente (sem custo) ou ter seu custo calculado a partir de valores médios. No sistema celular, a rede secundária pode ser associada à interface aérea da rede.

\subsection{Modelagem Matemática}

O modelo matemático é um problema de Programação Linear Inteira Mista (PLIM) com Variáveis 0-1, que utiliza a abordagem nó-arco. As variáveis de decisão referem-se ao valor do fluxo nos arcos e à alocação (ou não) e dimensionamento de facilidades (equipamentos de transmissão, cabos ópticos e metálicos, infra-estrutura) instaláveis em cada arco (ou nó) para o atendimento dos serviços. Apresenta a seguinte formulação (DeSousa et alii, 2003; Desousa et alii, 2001a):

$$
\operatorname{Maximizar} \mathrm{R}(\mathrm{y})=\sum_{(\mathrm{s}, \mathrm{i}) \in \mathrm{A}_{\mathrm{S}}} \mathrm{r}_{\mathrm{si}} Y_{\mathrm{si}}
$$

Sujeito a:

$$
\begin{aligned}
& \sum_{(\mathrm{i}, \mathrm{j}) \in \mathrm{A}_{\mathrm{ST}}} \sum_{\mathrm{n} \in \mathrm{N}_{\mathrm{STij}}}\left(\varphi_{\mathrm{ij}}^{X_{\mathrm{eq}}, \mathrm{n}}+\varphi_{\mathrm{ij}}^{X_{\mathrm{r}}, \mathrm{n}} \cdot l_{\mathrm{ij}}\right) \cdot X_{\mathrm{ijn}}+ \\
& \sum_{(\mathrm{i}, \mathrm{j}) \in \mathrm{A}_{\mathrm{M}}} \sum_{\mathrm{p} \in \mathrm{C}_{\mathrm{M}}} \varphi_{\mathrm{ij}}^{M, \mathrm{p}} \cdot M_{\mathrm{ijp}}+\sum_{(\mathrm{s}, \mathrm{i}) \in \mathrm{A}_{\mathrm{E}}} \varphi_{\mathrm{si}} \cdot Y_{\mathrm{si}} \leq \mathrm{L} \\
& \sum_{\mathrm{i} \in \mathrm{I}-\mathrm{I}_{\mathrm{S}}} Y_{\mathrm{si}}+Y e s c_{\mathrm{S}}=\mathrm{d}_{\mathrm{s}}, \forall \mathrm{s} \in \mathrm{I}_{\mathrm{S}} \\
& \sum_{\mathbf{j} \in \mathrm{J}_{1}} Y_{\mathrm{ij}}-\sum_{\mathrm{j} \in \mathrm{J}_{2}-\mathrm{I}_{\mathrm{S}}} Y_{\mathrm{ji}}-\sum_{\mathrm{s} \in \mathrm{I}_{\mathrm{S}}} \mathrm{fc}_{\mathrm{s}} \cdot Y_{\mathrm{si}}=0, \forall \mathrm{i} \in \mathrm{I}-\mathrm{I}_{\mathrm{S}} \\
& \sum_{\mathrm{n} \in \mathrm{N}_{\mathrm{STij}}} \operatorname{cap}_{\mathrm{ij}}^{X, \mathrm{n}} \cdot X_{\mathrm{ijn}} \geq Y_{\mathrm{ij}}, \forall(\mathrm{i}, \mathrm{j}) \in \mathrm{A}_{\mathrm{ST}} \\
& \sum_{\mathrm{p} \in \mathrm{C}_{\mathrm{M}}} \operatorname{cap}_{\mathrm{ij}}^{M, \mathrm{p}} \cdot M_{\mathrm{ijp}} \geq Y_{\mathrm{ij}}, \forall(\mathrm{i}, \mathrm{j}) \in \mathrm{A}_{\mathrm{M}}
\end{aligned}
$$

Onde:

$\mathbf{R}(\mathbf{y})$ : receita total dos serviços oferecidos;

$\mathbf{A}_{S}$ : conjunto de arcos artificiais de serviço (arcos de escoamento) que conectam os nós artificiais de serviço aos nós de acesso;

$\boldsymbol{Y}_{s i}$ : variável real não-negativa associada ao fluxo de demanda escoado pelo $\operatorname{arco}(\mathrm{s}, \mathrm{i}) \in \mathrm{A}_{S}$;

$\mathbf{r}_{s i}$ : receita unitária do serviço s oferecido ao nó de acesso i; 
$\mathbf{A}_{S T}$ : conjunto de arcos que podem receber como candidata a solução tecnológica $X$;

$\mathbf{N}_{S T i j}$ : conjunto de soluções tecnológicas candidatas no arco (i, j) $\in \mathrm{A}_{S T}$;

$\boldsymbol{X}_{i j n}$ : variável binária associada à escolha da solução tecnológica $X$, do tipo n, candidata no $\operatorname{arco}(i, j) \in \mathrm{A}_{S T}$;

$\varphi_{\mathrm{ij}}^{X_{\mathrm{eq}}, \mathrm{n}}:$ custo associado à escolha dos equipamentos da solução tecnológica $X$, do tipo $\mathrm{n}$, candidata no $\operatorname{arco}(\mathrm{i}, \mathrm{j}) \in$ $\mathrm{A}_{S T}$;

$\varphi_{\mathrm{ij}}^{X \mathrm{r}}, \mathrm{n}$ : custo (por unidade de comprimento) associado à escolha da infra-estrutura (cabos e dutos) da solução tecnológica $X$, do tipo $\mathrm{n}$, candidata no $\operatorname{arco}(\mathrm{i}, \mathrm{j}) \in \mathrm{A}_{S T}$;

$l_{i j}$ : comprimento do $\operatorname{arco}(\mathrm{i}, \mathrm{j}) \in \mathrm{A}_{S T}$;

$\mathbf{A}_{M}$ : conjunto de arcos que podem receber cabos metálicos;

$\mathbf{C}_{M}$ : conjunto de modularidades de cabos metálicos (novos);

$\boldsymbol{M}_{i j p}$ : variável binária associada à escolha do cabo metálico de modularidade $\mathrm{p}$, candidato no $\operatorname{arco}(\mathrm{i}, \mathrm{j}) \in \mathrm{A}_{M}$;

$\varphi_{\mathrm{ij}}^{M, \mathrm{p}}$ : custo associado à escolha do cabo metálico $M$, de modularidade $\mathrm{p}$, candidato no $\operatorname{arco}(\mathrm{i}, \mathrm{j}) \in \mathrm{A}_{M}$;

$\varphi_{\text {si }}$ : custo da rede secundária para a disponibilização do serviço do tipo s para o nó de acesso i, usando o $\operatorname{arco}(\mathrm{s}, \mathrm{i}) \in$ $\mathrm{A}_{S}$;

L : limitante de orçamento;

I :conjunto de todos os nós do grafo, exceto o nó da CC;

$\mathbf{I}_{S}$ : conjunto dos nós artificiais de serviço;

$\mathbf{J}_{1}$ : conjunto de nós $\mathrm{j}$ diretamente conectados ao nó $\mathrm{i}$, por arcos emanando de $\mathrm{i}$ para $\mathrm{j}$;

$\mathbf{J}_{2}$ : conjunto de nós j diretamente conectados ao nó i, por arcos emanando de $\mathrm{j}$ para $\mathrm{i}$;

Yesc $_{s}$ : variável real não-negativa associada à demanda não atendida do serviço s (fluxo através de um Arco de Escape);

$\mathbf{d}_{s}$ : demanda total do serviço $\mathrm{s}$, entrando na rede pelo nó $\mathrm{s} \in$ $\mathrm{I}_{S}$;

$\boldsymbol{Y}_{i j}$ : variável real não-negativa associada ao fluxo de demanda escoado pelo arco (i,j);

$\mathbf{f c}_{s}$ : fator de conversão de taxa de transmissão do serviço s; $\operatorname{cap}_{\mathrm{ij}}^{X, \mathrm{n}}$ : capacidade da solução tecnológica $X$, do tipo n, candidata no $\operatorname{arco}(\mathrm{i}, \mathrm{j}) \in \mathrm{A}_{S T}$;

$\operatorname{cap}_{\mathrm{ij}}^{M, \mathrm{p}}$ : capacidade do cabo metálico $M$, de modularidade $\mathrm{p}$, candidato (ou instalado) no $\operatorname{arco}(\mathrm{i}, \mathrm{j}) \in \mathrm{A}_{M}$;

Os principais componentes do modelo matemático são:

Função Objetivo (1): é a receita total, a ser maximizada;

Restrição de Limite de Orçamento (2): assegura que o custo total de alocação e dimensionamento dos equipamentos e infra-estrutura não ultrapasse o orçamento previsto. A primeira parcela da inequação refere-se ao custo de implantação da solução tecnológica X, a segunda aos custos com expansão em rede metálica e a última ao custo da rede secundária para a oferta dos serviços. Na aplicação do modelo, seja para o sistema de acesso fixo ou para a infra-estrutura do sistema de acesso celular, as parcelas são constituídas pelos custos de equipamentos e infra-estrutura candidatados pelo planejador;

Restrições de Satisfação de Demanda (3) e (4): garantem o balanço de fluxo em todos os nós do grafo, exceto para o nó da CC, por ser uma equação redundante. A restrição sobre o orçamento significa que parte da demanda pode não ser atendida. Por isso, são utilizados os Arcos de Escape. Devido à diversidade encontrada nas taxas de transmissão dos serviços, pode ser necessário utilizar alguns coeficientes de conversão $\left(\mathbf{f c}_{s}\right)$ na matriz de incidência;

Restrições Técnicas de Capacidade (5) e (6): ocorrem em cada arco previsto pelo planejador, assegurando que a soma das capacidades dos equipamentos implantados (ou alugados) seja superior ao fluxo escoado pelo arco. As especificações dos equipamentos candidatos depende do tipo de sistema de aceso em que se efetua a aplicação do modelo;

Restrições Adicionais: gerenciam o atendimento dos serviços (item 2.4). Outras restrições podem modelar também topologias e aspectos de segurança da rede, bem como funcionalidades e privilégios de alguns equipamentos.

\subsection{Controle de Atendimento dos Servi- ços}

A fim de assegurar preferências de atendimento, e ter um melhor controle da demanda a ser atendida, há a necessidade de se colocar limites para os fluxos a serem escoados pelos arcos do grafo. A seguir são apresentadas duas formas de efetuar este controle. 


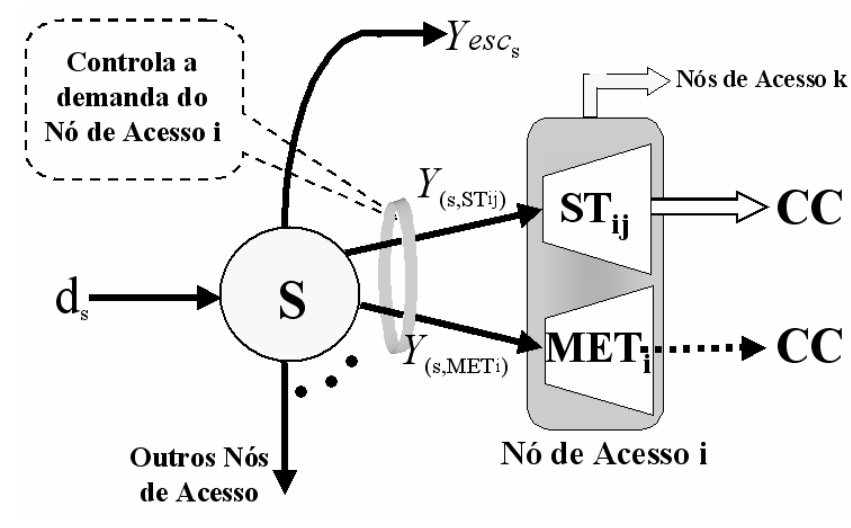

Figura 4: Controle de demanda por nó de acesso.

\subsubsection{Controle de Demanda por Nó de Acesso}

Este procedimento é útil quando se deseja controlar a demanda a ser atendida em cada Nó de Acesso, individualmente. Este controle é possível atribuindo-se limites aos $A r$ cos de Escoamento. A figura 4 e as equações (7) e (8) apresentam esta configuração de atendimento para um serviço $s$ em um Nó de Acesso $i$. A garantia de que o atendimento estará entre a demanda mínima $\left(d_{\min }{ }_{s i}\right)$ e máxima $\left(d \max _{s i}\right)$ é assegurada limitando-se a soma das demandas atendidas pela rede metálica $\left(M e t_{i}\right)$ e solução tecnológica $\left(S T_{i j}\right)$, em cada Nó de Acesso i. Nesta configuração, mesmo que um serviço não seja "rentável", garante-se, obrigatoriamente, o seu atendimento mínimo em cada região da rede atendida por um Nó de Acesso. Este controle torna-se interessante diante de contratos administrativos que exigem garantias de atendimento para uma determinada área ou um cliente em especial.

$$
\begin{gathered}
Y_{(\mathrm{s}, \mathrm{STij})}+Y_{(\mathrm{s}, \text { METi })} \geq \mathrm{dmin}_{\mathrm{si}}, \forall \mathrm{s} \in \mathrm{I}_{\mathrm{S}}, \forall \mathrm{i} \in \mathrm{I}-\mathrm{I}_{\mathrm{S}} \\
Y_{(\mathrm{s}, \mathrm{ST} \mathrm{ij})}+Y_{(\mathrm{s}, \text { METi })} \leq \operatorname{dmax}_{\mathrm{si}}, \forall \mathrm{s} \in \mathrm{I}_{\mathrm{S}}, \forall \mathrm{i} \in \mathrm{I}-\mathrm{I}_{\mathrm{S}}
\end{gathered}
$$

\subsubsection{Controle de Demanda por Serviço}

Este procedimento é utilizado quando se deseja garantir o atendimento do serviço, independente do Nó de Acesso. A demanda máxima prevista para cada Nó de Acesso $\left(\right.$ dmax $\left._{s i}\right)$ continua sendo controlada através dos Arcos de Escoamento. Já o atendimento mínimo deixa de ser gerenciado por Nó de Acesso e passa a ser assegurado apenas por serviço $\left(d_{m i n}\right)$, através da adoção de limites no Arco de Escape. A figura 5 e as equações (9) e (10) mostram este controle para um serviço $s$, bem como a forma de atendimento deste serviço em um Nó de Acesso i. A restrição no Arco de Escape define os limites para a demanda não atendida $\left(\operatorname{Yesc}_{s}\right)$, a qual atingirá no máximo a diferença entre a demanda total prevista

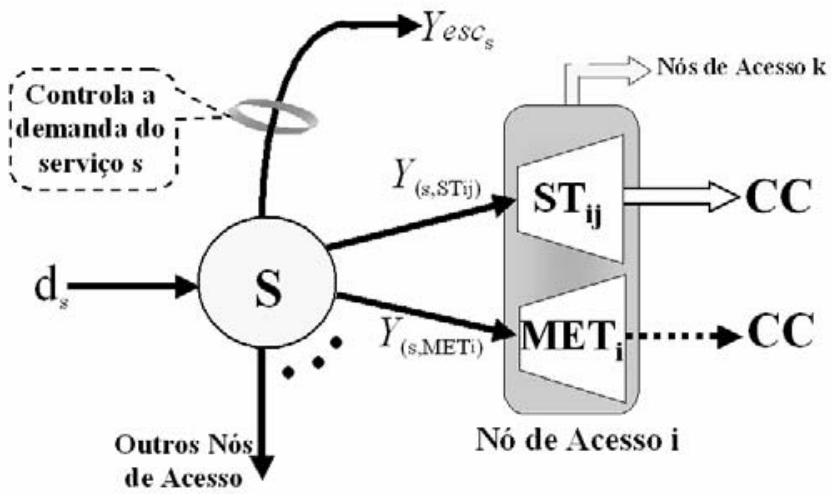

Figura 5: Controle de demanda por serviço.

$\left(d_{s}\right)$ e a demanda mínima a ser atendida $\left(d \min _{s}\right)$, garantindo o atendimento da demanda mínima para o serviço $s$. Neste contexto, como o objetivo do modelo é maximizar a receita gerada pelos serviços oferecidos, continua havendo a possibilidade de se escolher os Nós de Acesso mais "rentáveis", porém com a liberdade de até mesmo excluir (deixar de atender) os Nós de Acesso menos "rentáveis", fato proibido no Controle de Demanda por Nó de Acesso.

$$
\begin{gathered}
Y_{(\mathrm{s}, \mathrm{STij})}+Y_{(\mathrm{s}, \mathrm{METi})} \leq \operatorname{dmax}_{\mathrm{si}}, \forall \mathrm{s} \in \mathrm{I}_{\mathrm{S}}, \forall \mathrm{i} \in \mathrm{I}-\mathrm{I}_{\mathrm{S}} \\
Y e s c_{\mathrm{S}} \leq\left(\mathrm{d}_{\mathrm{S}}-\operatorname{dmin}_{\mathrm{S}}\right), \forall \mathrm{s} \in \mathrm{I}_{\mathrm{S}}
\end{gathered}
$$

\subsection{Adaptações para o Planejamento a Mínimo Custo}

Na abordagem de minimização de custo tem-se como objetivo principal dimensionar o sistema de acesso da forma mais barata possível para atender uma demanda fixa préestabelecida. Para que o modelo de maximização de receita seja capaz de realizar o dimensionamento nestas circunstâncias são necessárias pequenas adaptações:

- A função objetivo (1) deve ser substituída pela restrição (2). Esta expressão continua contabilizando os custos com a rede a ser implantada, porém com o objetivo de escolher a mais barata, e não mais aquela que respeite um orçamento;

- As restrições de satisfação de demanda (3) e (4) e as restrições técnicas de capacidade (5) e (6) continuam as mesmas, sem nenhuma modificação;

- Uma vez que toda a demanda apresentada aos nós artificiais de serviço deve ser atendida, basta proibir o fluxo desta demanda nos arcos de escape de cada ser-

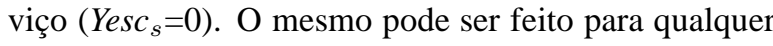
outro nível de atendimento de demanda. 


\section{MODELAGEM PARA A IMPRECISÃO NOS DADOS DE DEMANDA}

A demanda é quantificada em termos do número de assinantes de um ou mais serviços, e representa um dado de entrada para o modelo exato. A penetração do serviço de VOZ já é um fato conhecido, o que garante uma certa maturidade sobre o planejamento da rede. Porém, num ambiente com propostas de novos serviços esta informação nem sempre é precisa e encontra-se disponível. Uma vez que a variação neste parâmetro afeta o dimensionamento dos equipamentos exigidos e, consequentemente, os custos de implantação do sistema, esta informação representa um ponto crítico no processo de decisão. Neste sentido, o modelo apresentado anteriormente precisa ser modificado a fim de acomodar a possibilidade de avaliação dos riscos inerentes ao planejamento sob condições de imprecisão para os níveis de demada dos serviços.

Numa situação típica, o planejador possui uma boa idéia a respeito do grau de penetração do serviço, ou seja, ele é capaz de definir, para o número total de usuários de uma área de atendimento, uma faixa de possíveis valores para a demanda deste ou aquele serviço, inclusive com discriminação de valores com maior ou menor possibilidade de ocorrência. Esta particularidade quanto aos dados de demanda sugere a adoção do conceito de número fuzzy (Pedrycz e Gomide, 1998) para representá-los. Uma outra abordagem, utilizando a teoria de probabilidades, pode ser encontrada em (Ims et alii., 1997).

\subsection{Representação da Demanda Impre- cisa por Números Fuzzy}

A cada um dos níveis de demanda dos serviços é associado um conjunto fuzzy $\tilde{\mathrm{d}}_{\mathrm{si}}=\left(\underline{\mathrm{d}}_{\mathrm{si}}, \mathrm{D}_{\mathrm{si}}, \overline{\mathrm{d}}_{\mathrm{si}}\right)$, definido como "o conjunto dos valores possíveis para a demanda do serviço $s \in \mathrm{I}_{S}$ a ser atendida no nó de acesso $i \in \mathrm{I}$ ". É adotada uma função de pertinência triangular. A figura 6 apresenta o número fuzzy resultante desta definição, onde $\mathbf{D}_{s i}$ é o valor de maior pertinência.

No modelo, as adaptações ocorrem nas restrições (3), (7), (8), que é idêntica à (9), e (10), que passam a ser, respectivamente (DeSousa e Carlson, 2000):

$$
\begin{aligned}
& \sum_{\mathrm{i} \in \mathrm{I}-\mathrm{I}_{\mathrm{S}}} Y_{\mathrm{si}}+Y e s c_{\mathrm{S}}=\tilde{\mathrm{d}}_{\mathrm{S}}, \forall \mathrm{s} \in \mathrm{I}_{\mathrm{S}} \\
& Y_{(\mathrm{s}, \mathrm{STij})}+Y_{(\mathrm{s}, \mathrm{METi})} \geq \tilde{\mathrm{d}} \mathrm{min}_{\mathrm{si}}, \forall \mathrm{s} \in \mathrm{I}_{\mathrm{S}}, \forall \mathrm{i} \in \mathrm{I}-\mathrm{I}_{\mathrm{S}} \\
& Y_{(\mathrm{s}, \mathrm{STij})}+Y_{(\mathrm{s}, \mathrm{METi})} \leq \tilde{\mathrm{d}}_{\mathrm{max}_{\mathrm{Si}}}, \forall \mathrm{s} \in \mathrm{I}_{\mathrm{S}}, \forall \mathrm{i} \in \mathrm{I}-\mathrm{I}_{\mathrm{S}} \\
& Y e s c_{\mathrm{s}} \leq\left(\tilde{\mathrm{d}}_{\mathrm{s}}-\tilde{\mathrm{d}} \min _{\mathrm{s}}\right), \forall \mathrm{s} \in \mathrm{I}_{\mathrm{S}}
\end{aligned}
$$

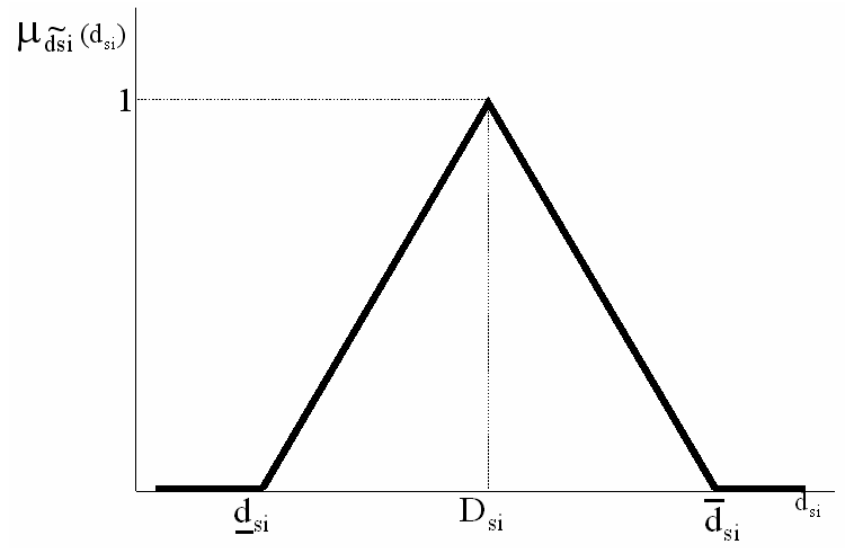

Figura 6: Representação da demanda imprecisa.

As variáveis e os parâmetros das restrições (11)-(14) são os mesmos descritos no item 2.3, enquanto os termos independentes são representados por dados imprecisos:

$\tilde{\mathrm{d}}_{\mathrm{S}}:$ demanda total (imprecisa) do serviço s, entrando na rede pelo nó $\mathrm{s} \in \mathrm{I}_{S}$;

$\tilde{\mathrm{d}} \min _{\mathrm{si}}$ : demanda mínima prevista (imprecisa) para o serviço s, no nó de acesso $\mathrm{i} \in \mathrm{I}_{-} \mathrm{I}_{S}$;

$\tilde{\mathrm{dmax}}_{\mathrm{Si}}$ : demanda máxima prevista (imprecisa) para o serviço s, no nó de acesso $\mathrm{i} \in \mathrm{I}-\mathrm{I}_{S}$;

$\tilde{\mathrm{d}} \min _{\mathrm{S}}$ : demanda mínima (imprecisa) do serviço s, a ser atendida pela rede, entrando pelo nó $\mathrm{i} \in \mathrm{I}_{S}$.

Portanto, a definição da demanda prevista (imprecisa) em cada nó da rede (nós de serviço e nós de acesso) é obtida efetuando-se operações com números fuzzy (adição, subtração e multiplicação por escalar).

\subsection{Resolução do Modelo Fuzzy}

A presença de números fuzzy nas restrições do modelo pode alterar substancialmente o procedimento de resolução. Há a necessidade de se transformar os números fuzzy de maneira a permitir o seu tratamento.

Neste trabalho optou-se pela substituição do número fuzzy por um parâmetro que permita um processo de resolução mais simples sem perder as características de imprecisão expressas pelo número fuzzy. A idéia é parametrizar o substituto do número fuzzy, visando o tratamento do problema sem prescindir da imprecisão sobre os dados usados. No nosso caso, a variação do parâmetro permite analisar diferentes possibilidades para o valor assumido como demanda dos serviços. A solução do problema é dependente do valor do parâmetro. 


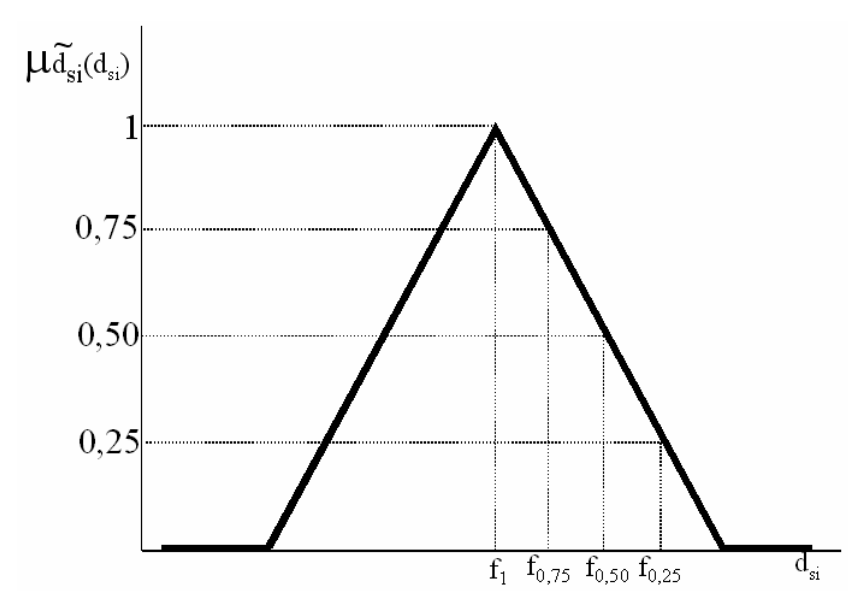

Figura 7: Equivalente de Adamo para um número fuzzy triangular.

A função paramétrica adotada para a determinação do equivalente do número fuzzy é a de Adamo (Campos e Verdegay, 1989):

$$
\mathrm{f} \alpha\left(\tilde{\mathrm{d}}_{\mathrm{si}}\right)=\max \left\{\mathrm{d}_{\mathrm{si}} \mid \mu \tilde{\mathrm{d}}_{\mathrm{si}}\left(\mathrm{d}_{\mathrm{si}}\right) \geq \alpha\right\}
$$

$\operatorname{com} \alpha \in[0,1]$.

Para o caso triangular tem-se:

$$
\mathrm{f} \alpha\left(\tilde{\mathrm{d}}_{\mathrm{si}}\right)=\mathrm{D}_{\mathrm{si}}+\left(\overline{\mathrm{d}}_{\mathrm{si}}-\mathrm{D}_{\mathrm{si}}\right) \cdot(1-\alpha)
$$

O número fuzzy é reduzido a um intervalo do qual se toma o limitante superior como valor de trabalho. O parâmetro $\alpha$ indica o grau de confiança nos valores a adotar. A figura 7 mostra o funcionamento do equivalente de Adamo para um número fuzzy triangular.

Mantendo-se a linearidade da função de Adamo para números fuzzy triangulares, as restrições (11), (12) e (13) e (14) tornam-se, respectivamente:

$$
\sum_{\mathrm{i} \in \mathrm{I}-\mathrm{I}_{\mathrm{S}}} Y_{\mathrm{Si}}+Y e s c_{\mathrm{S}}=\mathrm{D}_{\mathrm{S}}+\left(\overline{\mathrm{d}}_{\mathrm{S}}-\mathrm{D}_{\mathrm{S}}\right) \cdot(1-\alpha), \forall \mathrm{s} \in \mathrm{I}_{\mathrm{S}}
$$

$$
\begin{aligned}
& Y_{(\mathrm{s}, \mathrm{STij})}+Y_{(\mathrm{s}, \mathrm{METi})} \geq \operatorname{Dmin}_{\mathrm{si}}+ \\
& \left(\overline{\mathrm{dmin}}_{\mathrm{si}}-\operatorname{Dmin}_{\mathrm{si}}\right) \cdot(1-\alpha), \forall \mathrm{s} \in \mathrm{I}_{\mathrm{S}}, \forall \mathrm{i} \in \mathrm{I}-\mathrm{I}_{\mathrm{S}} \\
& Y_{(\mathrm{s}, \mathrm{STij})}+Y_{(\mathrm{s}, \mathrm{METi})} \leq \operatorname{Dmax}_{\mathrm{si}}+ \\
& \left(\overline{\mathrm{dmax}}_{\mathrm{Si}}-\operatorname{Dmax}_{\mathrm{si}}\right) \cdot(1-\alpha), \forall \mathrm{s} \in \mathrm{I}_{\mathrm{S}}, \forall \mathrm{i} \in \mathrm{I}-\mathrm{I}_{\mathrm{S}}
\end{aligned}
$$

$$
\begin{aligned}
& Y e s c_{\mathrm{S}} \leq\left\{\left[\mathrm{D}_{\mathrm{S}}+\left(\overline{\mathrm{d}}_{\mathrm{S}}-\mathrm{D}_{\mathrm{S}}\right) \cdot(1-\alpha)\right]-\right. \\
& \left.\left[\operatorname{Dmin}_{\mathrm{S}}+\left(\overline{\mathrm{d} m i n} \mathrm{Dm}_{\mathrm{S}}-\operatorname{Dmin}_{\mathrm{S}}\right) \cdot(1-\alpha)\right]\right\}, \forall \mathrm{s} \in \mathrm{I}_{\mathrm{S}}
\end{aligned}
$$

A variação do parâmetro $\alpha$ permite fazer uma análise técnico-econômica para diferentes possibilidades de valor de demanda prevista de cada serviço, em cada nó de acesso. Outro fato a ser observado é que a demanda dos serviços nem sempre apresentará valores inteiros, o que pode parecer um pouco estranho. Embora seja possível forçar estes valores a serem inteiros, o que acarreta um processamento mais "pesado"para o problema, este procedimento é desnecessário neste nível de planejamento. Uma alternativa interessante, e também sem perda de generalidade, seria adotar valores arredondados (ou truncados) para a demanda resultante da aplicação do parâmetro $\alpha$.

Neste mesmo contexto, a avaliação da demanda imprecisa segundo uma ótica multi-paramétrica (diferentes níveis de confiança - $\alpha_{s i}$ - para os valores de demanda de cada serviço, em regiões distintas da rede) também poderia ser adicionada ao modelo, porém a base de dados aumentaria consideravelmente. Outro agravante é o fato de que o procedimento de resolução para obter a solução completa do modelo multi-paramétrico pode-se tornar consideravelmente mais complexo (Crema, 2002).

\section{EXEMPLOS DE APLICAÇÃO: SISTEMA DE ACESSO CELULAR}

Vários cenários de planejamento para a infra-estrutura do sistema de acesso móvel celular foram estudados com o objetivo de avaliar o desempenho do modelo. A avaliação do risco técnico-econômico é realizada com o objetivo de medir o impacto sofrido pelo sistema em situações de variações de orçamento e imprecisão nos dados de demanda dos serviços. Em ambos os casos são analisados os dois tipos de controle de demanda: por nó de acesso e por serviço.

As principais variáveis a serem analisadas são: hierarquia dos equipamentos a serem alocados, topologia da rede, demanda a ser atendida de cada serviço, em cada nó de acesso e o comportamento da receita gerada pelo sistema.

Todos os experimentos computacionais foram realizados em uma máquina Sun Workstation Ultra-1 com sistema operacional SunOS 5.7. As instâncias foram geradas em linguagem C e resolvidas com o solver CPLEX (1994).

Uma aplicação da metodologia à infra-estrutura do sistema de acesso celular, com a abordagem de minimização de custo, pode ser conferida em DeSousa et alii (2001). DeSousa et alii (2001b) apresenta aplicações da metodologia ao sistema de acesso fixo, onde são avaliadas as tecnologias xDSL e FITL, com a oferta de quatro tipos de serviços. 


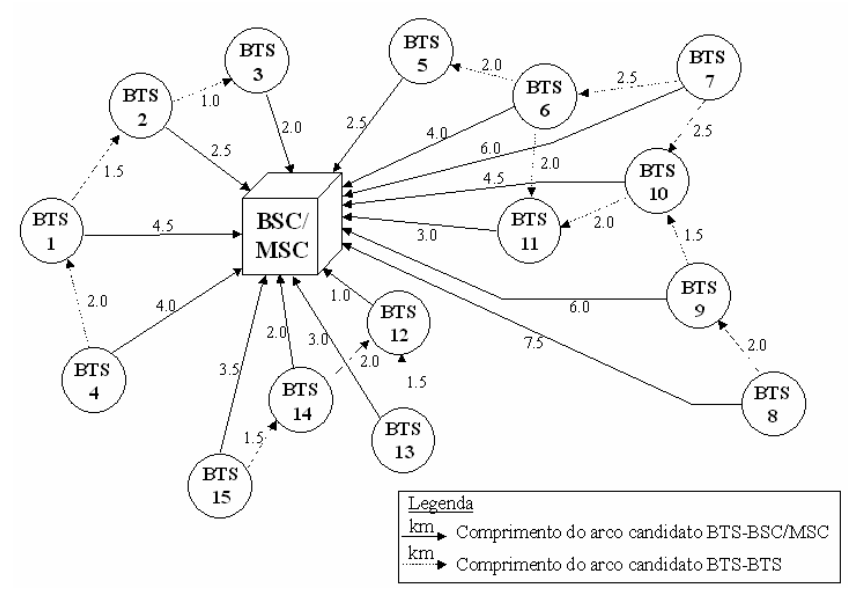

Figura 8: Rede analisada.

\begin{tabular}{|l|c|c|c|c|c|c|}
\hline \multirow{2}{*}{$\begin{array}{l}\text { Sistema } \\
\text { candidato }\end{array}$} & \multicolumn{5}{|c|}{ Custos } & $\begin{array}{c}\text { Infra-estrutura } \\
\text { Equipamentos }\end{array}$ \\
\cline { 2 - 7 } & $1 \times E 1$ & $2 \times E 1$ & $4 \times E 1$ & $8 \times E 1$ & Dutos & Cabos \\
\cline { 2 - 7 } & 1,00 & - & - & - & 4,17 & 4,30 \\
\hline HDSL & 1,00 & 1,50 & 1,75 & 2,00 & 5,00 & 3,80 \\
\hline Óptico & - & 8,50 & 13,50 & 20,00 & 0 & 0 \\
\hline Rádio & & \multicolumn{7}{|c|}{} &
\end{tabular}

Tabela 2: Informações sobre as tecnologias.

\subsection{Dados de Entrada para o Modelo}

Os parâmetros do modelo foram escolhidos de forma a refletir dados reais tanto quanto possível. A figura 8 mostra a rede exemplo, com 1 BSC/MSC e 15 BTS, bem como o comprimento dos arcos candidatos. A demanda em cada nó pode ser atendida através de um arco direto (BTS-BSC/MSC) e/ou utilizar uma (ou mais) rota alternativa (BTS-BTS).

Três tecnologias de transmissão foram consideradas como candidatas: modems HDSL (High-data-rate Digital Subscriber Line), modems ópticos e enlaces via rádio. As três apresentam taxa de transmissão simétrica. A tabela 2 lista as capacidades (em canais E1) e os custos de equipamentos/infraestrutura para cada tecnologia. O custo do sistema HDSL é adotado como referência. A distância máxima permitida para um enlace com HDSL é de $4 \mathrm{~km}$.

A rede, utilizando as três tecnologias candidatas, é capaz de oferecer dois perfis de serviço, um que exige canais de transmissão a 64 kbps e outro a 144 kbps, ambos simétricos e com comutação por circuito. A demanda (imprecisa) é indicada por números fuzzy triangulares (tabela 3 ). Os triângulos são todos simétricos, embora isto não seja obrigatório. O parâmetro de confiabilidade $\alpha$, de cada serviço, é o mesmo para

\begin{tabular}{|c|c|c|c|c|c|c|}
\hline \multirow{3}{*}{ BTS } & \multicolumn{6}{|c|}{ Demanda (em número de canais) } \\
\hline & \multicolumn{3}{|c|}{ Serviço 01} & \multicolumn{3}{|c|}{ Serviço 02} \\
\hline & $\underline{\mathbf{d}}_{s i}$ & $\overline{\mathbf{D}_{s i}}$ & $\overline{\bar{d}}_{s i}$ & $\underline{\mathbf{d}}_{s i}$ & $\overline{\mathbf{D}_{s i}}$ & $\overline{\bar{d}}_{s i}$ \\
\hline 01 & 11 & 18 & 25 & 07 & 13 & 19 \\
\hline 02 & 10 & 21 & 32 & 06 & 11 & 16 \\
\hline 03 & 10 & 15 & 20 & 05 & 09 & 13 \\
\hline 04 & 08 & 16 & 24 & 07 & 12 & 17 \\
\hline 05 & 12 & 18 & 24 & 10 & 15 & 20 \\
\hline 06 & 16 & 23 & 30 & 04 & 12 & 20 \\
\hline 07 & 18 & 26 & 34 & 06 & 10 & 14 \\
\hline 08 & 09 & 14 & 19 & 05 & 09 & 13 \\
\hline 09 & 13 & 20 & 27 & 07 & 11 & 15 \\
\hline 10 & 18 & 24 & 30 & 09 & 12 & 15 \\
\hline 11 & 11 & 20 & 29 & 06 & 10 & 14 \\
\hline 12 & 13 & 18 & 23 & 07 & 12 & 17 \\
\hline 13 & 13 & 19 & 25 & 05 & 10 & 15 \\
\hline 14 & 07 & 16 & 25 & 04 & 08 & 12 \\
\hline 15 & 08 & 14 & 20 & 11 & 14 & 17 \\
\hline Total & 177 & 282 & 387 & 99 & 168 & 237 \\
\hline \multicolumn{7}{|c|}{ Atendimento mínimo (\%) } \\
\hline & \multicolumn{3}{|c|}{50} & \multicolumn{3}{|c|}{50} \\
\hline \multicolumn{7}{|c|}{ Receita } \\
\hline & \multicolumn{3}{|c|}{0.1} & \multicolumn{3}{|c|}{0.2} \\
\hline
\end{tabular}

Tabela 3: Informações sobre os serviços.

todos os nós de acesso. A tabela 3 também lista o fator de atendimento mínimo e a receita unitária (valor relativo) para os dois serviços considerados.

\subsection{Adaptações no Modelo}

Para a aplicação da modelagem apresentada nos ítens 2 e 3, existe a necessidade de efetuar algumas adaptações nas restrições referentes à representação das tecnologias candidatas. Isto acontece em função de especificidades referentes a limitações técnicas, como por exemplo o comprimento máximo de um enlace HDSL, ou a garantia de melhor utilização ao estimular (ou proibir) a confirguração de uma determinado tipo de topologia.

Para as tecnologias consideradas no planejamento da infraestrutrura do sistema celular são necessárias as seguintes adaptações (DeSousa et alii, 2003a):

- Substituir a restrição (2), que limita o orçamento, pela restrição (21): 


$$
\begin{gathered}
\sum_{(\mathrm{i}, \mathrm{j}) \in \mathrm{A}} \sum_{\mathrm{n} \in \mathrm{H}_{\mathrm{ij}}}\left[\left(\varphi_{\mathrm{ij}}^{\mathrm{H}_{\mathrm{eq}}, \mathrm{n}}+\varphi_{\mathrm{ij}}^{\mathrm{H}_{\mathrm{r}}, \mathrm{n}} \cdot l_{\mathrm{ij}}\right) \cdot \sum_{\mathrm{k} \in \psi} 2^{\mathrm{k}} \cdot X_{\mathrm{ijnk}}\right]+ \\
\sum_{(\mathrm{i}, \mathrm{j}) \in \mathrm{A}} \sum_{\mathrm{n} \in \mathrm{O}_{\mathrm{ij}}}\left(\varphi_{\mathrm{ij}}^{\mathrm{O}_{\mathrm{eq}}, \mathrm{n}}+\varphi_{\mathrm{ij}}^{\mathrm{O}_{\mathrm{r}}, \mathrm{n}} \cdot l_{\mathrm{ij}}\right) \cdot Z_{\mathrm{ijn}}+ \\
\left.\sum_{(\mathrm{s}, \mathrm{i}) \in \mathrm{An} \in \mathrm{R}_{\mathrm{ij}}} \sum_{\left(\varphi_{\mathrm{ij}}^{\mathrm{R}}\right.}^{\mathrm{R}_{\mathrm{eq}}, \mathrm{n}}+\varphi_{\mathrm{ij}}^{\mathrm{R}}, l_{\mathrm{ij}}, \mathrm{n}\right) \cdot W_{\mathrm{ijn}} \leq \mathrm{L}
\end{gathered}
$$

Onde:

A : conjunto de arcos conectanto nós BTS-BTS ou nós BTSBSC/MSC;

$\mathbf{H}_{i j}$ : conjunto de sistemas de modems HDSL candidatos no $\operatorname{arco}(\mathrm{i}, \mathrm{j}) \in \mathrm{A}$;

$\psi$ : conjunto dos números naturais não-negativos $\{0,1,2,3$ $\ldots\}$;

$\boldsymbol{X}_{i j n k}$ : variável binária, de ordem k, associada à escolha do sistema de modems HDSL, tipo $n$, candidato no $\operatorname{arco}(i, j) \in$ A;

$\varphi_{\mathrm{ij}}^{\mathrm{Heq}, \mathrm{n}}$ : custo do sistema de modems HDSL (equipamentos), tipo $\mathrm{n}$, candidato no $\operatorname{arco}(\mathrm{i}, \mathrm{j}) \in \mathrm{A}$;

$\varphi_{\mathrm{ij}}^{\mathrm{Hr}, \mathrm{n}}$ : custo do sistema de modems HDSL (cabos e dutos), tipo $\mathrm{n}$, candidato no $\operatorname{arco}(\mathrm{i}, \mathrm{j}) \in \mathrm{A}$;

$\mathbf{O}_{i j}$ : conjunto dos sistemas de modems ópticos candidatos no $\operatorname{arco}(i, j) \in A$;

$\boldsymbol{Z}_{i j n}$ : variável binária associada à escolha do sistema de modem ópticos, tipo $n$, candidato no arco $(\mathrm{i}, \mathrm{j}) \in \mathrm{A}$;

$\varphi_{\mathrm{ij}}^{\mathrm{Oeq}, \mathrm{n}}$ : custo do sistema de modems ópticos (equipamentos), tipo $\mathrm{n}$, candidato no $\operatorname{arco}(\mathrm{i}, \mathrm{j}) \in \mathrm{A}$;

$\varphi_{\mathrm{ij}}^{\mathrm{Or}, \mathrm{n}}$ : custo do sistema de modems ópticos (cabos e dutos), tipo $\mathrm{n}$, candidato no $\operatorname{arco}(\mathrm{i}, \mathrm{j}) \in \mathrm{A}$;

$\mathbf{R}_{i j}$ : conjunto dos sistemas rádio candidatos no $\operatorname{arco}(\mathrm{i}, \mathrm{j}) \in$ A;

$\boldsymbol{W}_{i j n}$ : variável binária associada à escolha do sistema rádio, tipo $\mathrm{n}$, candidato no $\operatorname{arco}(\mathrm{i}, \mathrm{j}) \in \mathrm{A}$;

$\varphi_{\mathrm{ij}}^{\mathrm{Req}, \mathrm{n}}$ : custo do sistema rádio (equipamentos), tipo n, candidato no $\operatorname{arco}(i, j) \in A$;
$\varphi_{\mathrm{ij}}^{\mathrm{Rr}, \mathrm{n}}$ : custo do sistema rádio (repetidores e amplificadores), tipo $n$, candidato no $\operatorname{arco}(\mathrm{i}, \mathrm{j}) \in \mathrm{A}$;

$\boldsymbol{l}_{i j}:$ comprimento do $\operatorname{arco}(\mathrm{i}, \mathrm{j}) \in \mathrm{A}$.

- Substituir a restrição (5), que controla a capacidade das tecnologias escolhidas, pela restrição (22):

$$
\begin{gathered}
\sum_{\mathrm{n} \in \mathrm{H}_{\mathrm{ij}}}\left[\operatorname{cap}_{\mathrm{ij}}^{X, \mathrm{n}} \cdot \sum_{\mathrm{k} \in \psi} 2^{\mathrm{k}} \cdot X_{\mathrm{ijnk}}\right]+\sum_{\mathrm{n} \in \mathrm{M}_{\mathrm{ij}}} \operatorname{cap}_{\mathrm{ij}}^{Z, \mathrm{n}} \cdot Z_{\mathrm{ijn}}+ \\
\sum_{\mathrm{n} \in \mathrm{R}_{\mathrm{ij}}} \operatorname{cap}_{\mathrm{ij}}^{W, \mathrm{n}} \cdot W_{\mathrm{ijn}} \geq Y_{\mathrm{ij}} \geq 0, \forall(\mathrm{i}, \mathrm{j}) \in \mathrm{A}
\end{gathered}
$$

Onde:

$\operatorname{cap}_{\mathrm{ij}}^{X, \mathrm{n}}$ : capacidade do sistema de modems HDSL, tipo n, candidato no $\operatorname{arco}(i, j) \in A$;

$\operatorname{cap}_{\mathrm{ij}}^{Z, \mathrm{n}}$ : capacidade do sistema de modems ópticos, tipo n, candidato no $\operatorname{arco}(i, j) \in A$;

$\operatorname{cap}_{\mathrm{ij}}^{W, \mathrm{n}}$ : capacidade do sistema rádio, tipo $\mathrm{n}$, candidato no $\operatorname{arco}(i, j) \in A$.

- Acrescentar a restrição (23), para garantir que a escolha da tecnologia HDSL só aconteça para aqueles enlaces com comprimento no máximo igual ao permitido:

$$
X_{\mathrm{ijnk}}=0, \forall l_{\mathrm{ij}}>l_{\max }
$$

Onde:

$l_{\text {max }}$ : comprimento máximo permitido para um enlace HDSL.

- Acrescentar a restrição (24) para proibir a formação de rotas alternativas onde se tenha o compartilhamento de um enlace utilizando sistemas HDSL:

$$
\begin{array}{r}
\sum_{\mathbf{j} \in \mathrm{J}_{1}} \sum_{\mathrm{n} \in \mathrm{H}_{\mathrm{ij}}}\left[\operatorname{cap}_{\mathrm{ij}}^{X, \mathrm{n}} \cdot \sum_{\mathrm{k} \in \psi} 2^{\mathrm{k}} \cdot X_{\mathrm{ijnk}}\right]- \\
\sum_{\mathrm{s} \in \mathrm{I}_{\mathrm{S}}} \mathrm{fc}_{\mathrm{s}} \cdot Y_{\mathrm{si}} \leq 0, \forall \mathrm{i} \in \mathrm{I}-\mathrm{I}_{\mathrm{s}}
\end{array}
$$




\subsection{Aplicação do Modelo Exato: Impacto da Variação de Orçamento}

Nesta análise foi verificado o impacto causado por variações no orçamento disponível para investimento no sistema. A configuração de atendimento (rede e serviços) e os indicadores de receita são apresentados.

Foi considerado, para a rede da figura 8, que apenas os dutos dos sistemas HDSL e dos modems ópticos já estavam instalados. As tecnologias candidatas são aquelas apresentadas na tabela 2. Optou-se por avaliar o cenário mais otimista, com os valores de demanda sendo fixados em seus valores máximos $\left(\bar{d}_{s i}\right.$, tabela 3$)$. A análise para os outros dois níveis de demanda pode ser feita seguindo o mesmo procedimento adotado aqui.

O planejamento do sistema foi feito, primeiramente, adotando-se o critério de minimização de custo. Nestas condições, é necessário um orçamento de 175,60 unidades de custo, capaz de implantar a rede mostrada na figura 9, que atende toda a demanda prevista. Essa demanda produz 86,10 unidades de receita.

Diante da possibilidade do orçamento disponível ser inferior ao necessário para o atendimento de toda a demanda, o modelo de maximização é utilizado para obter configurações de rede que garantam as maiores receitas. As possíveis alterações de uma instância para outra são causadas, principalmente, pela forma de atendimento dos serviços e pela tecnologia adotada.

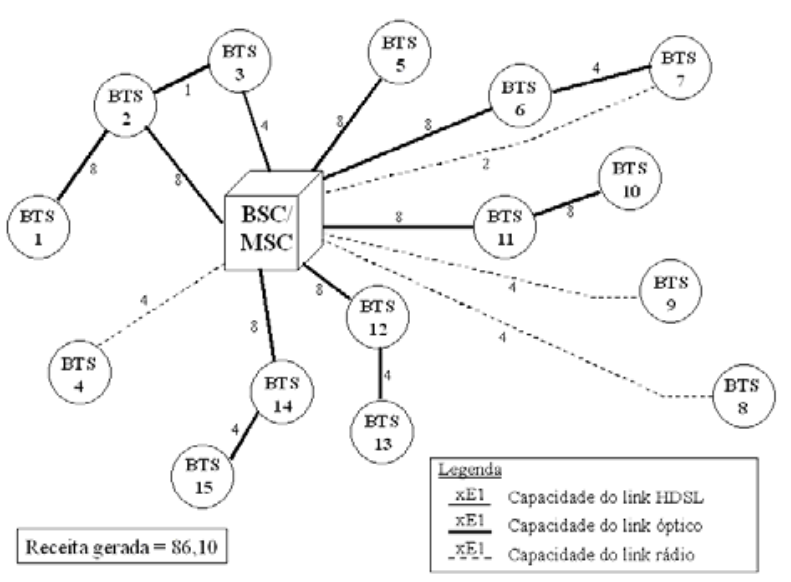

Figura 9: Configuração da rede para atendimento de toda a demanda (solução de mínimo custo).

\begin{tabular}{|c|c|c|c|c|c|}
\hline & \multicolumn{5}{|c|}{$\begin{array}{c}\text { Previsão de orçamento } \\
\text { (porcentagem do total 175,6) }\end{array}$} \\
\hline & $100 \%$ & $95 \%$ & $90 \%$ & $85 \%$ & $80 \%$ \\
\hline \multicolumn{6}{|c|}{ Receita gerada } \\
\hline & 86,10 & 85,39 & 83,68 & 77,24 & 71,69 \\
\hline \multicolumn{6}{|c|}{ Receita / custo } \\
\hline & 0,49 & 0,51 & 0,53 & 0,52 & 0,51 \\
\hline \multicolumn{6}{|c|}{ Número de canais não atendidos } \\
\hline Serviço 1 & 0 & 0 & 0 & 21,50 & 24,50 \\
\hline Serviço 2 & 0 & 3,55 & 12,11 & 33,56 & 59,78 \\
\hline \multicolumn{6}{|c|}{ Sistemas escolhidos (x E1) } \\
\hline HDSL & 0 & 0 & 0 & 0 & 0 \\
\hline $\begin{array}{l}\text { Óptico } \\
(1 / 2 / 4 / 8)\end{array}$ & $1 / 0 / 4 / 8$ & $0 / 0 / 3 / 9$ & $0 / 0 / 2 / 8$ & $0 / 0 / 5 / 5$ & $0 / 0 / 3 / 9$ \\
\hline $\begin{array}{l}\text { Rádio } \\
(2 / 4 / 8)\end{array}$ & $1 / 3 / 0$ & $0 / 3 / 0$ & $0 / 5 / 0$ & $3 / 2 / 0$ & $1 / 2 / 0$ \\
\hline
\end{tabular}

Tabela 4: Soluções para o cenário do item 4.3.1.

\subsubsection{Análise Técnico-Econômica pelo Controle de Demanda por Nó de Acesso}

Aqui foi respeitado o atendimento mínimo (tabela 3) em cada BTS, controlando os limites de demanda em cada célula do sistema de acesso móvel celular. Os resultados para diferentes condições de orçamento são apresentados na tabela 4. As receitas geradas e as configurações de atendimento são apresentadas como valores gerais, mas podem ser facilmente detalhadas, como, por exemplo, receita por serviço, custo por tecnologia e tecnologia/hierarquia por enlace escolhido. Através de simulações foi confirmado que uma queda de $25 \%$ no orçamento torna inviável a obtenção de uma solução factível para o problema.

Para os dados considerados, pode-se observar que os sistemas de modems ópticos são predominantes em todas as instâncias. Em valores absolutos, o serviço 2 foi o mais penalizado. Embora aconteça reduções de orçamento, a relação receita/custo mantém-se estável, ou seja, mesmo que não haja condição financeira de atender todos os usuários previstos, as redes projetadas garantem um retorno em torno de $50 \%$ do capital investido.

A configuração da rede para a previsão de investimento de $80 \%$ do orçamento total pode ser vista na figura 10. Apenas seis BTSs $(4,6,12,13,14$ e 15) não apresentaram demanda reprimida. Pode-se observar também que as maiores perdas de receita ocorreram nas BTSs 3 e 11. Embora o atendimento, para os dois serviços, tenha ficado próximo do mínimo nestas duas BTSs, elas possuem uma localização es- 


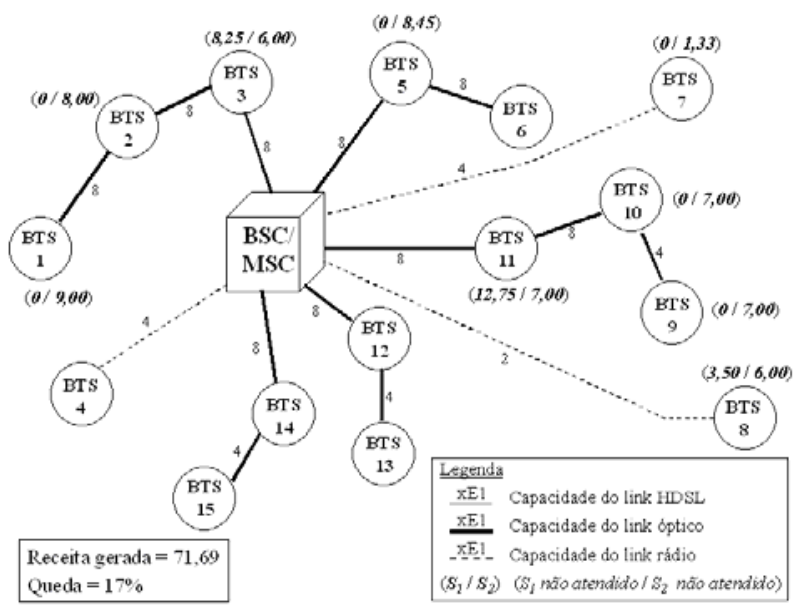

Figura 10: Configuração da rede para o cenário do item 4.3.1.

tratégica muito importante dentro da configuração escolhida, uma vez que elas agregam demandas oriundas de outras regiões da rede.

\subsubsection{Análise Técnico-Econômica pelo Controle de Demanda por Serviço}

Aqui foi respeitado o atendimento mínimo (tabela 3) por serviço, controlando os limites de demanda na área total atendida por uma BSC/MSC. São consideradas as mesmas condições de rede instalada e serviços previstos do item 4.3.1. O orçamento necessário para atender toda a demanda continua sendo 175,60. A rede também foi dimensionada para diferentes valores de orçamento. Os resultados são listados na tabela 5. Para este cenário, a infactibilidade acontece a partir de uma queda de $61 \%$ no orçamento.

À medida que o orçamento fica mais enxuto, o serviço 1 é o que sofre as maiores perdas na demanda atendida. Para as previsões de orçamento mais pessimistas, tem-se, inclusive, a escolha de não se alocar equipamentos em algumas BTSs. As BTSs 8 e 9 apresentam-se como as menos atrativas. Em termos econômicos, pode-se observar que, caso aconteça uma redução de $50 \%$ no orçamento, a receita fica penalizada em $40 \%$, enquanto a relação receita/custo tem um aumento de $20 \%$ (de 0,49 para 0,59). Em termos de receita, pode-se observar também que a não-exigência de atendimento mínimo em determinados setores da rede possibilita um planejamento com maior retorno financeiro, por exemplo: para uma mesma previsão de orçamento ( $80 \%$ ), tem-se que a exigência de demanda mínima em cada BTS (cenário 4.3.1) faz com que a receita caia de 76,58 para 71,69 (perda de $6,5 \%$ ).

A figura 11 mostra a configuração da rede para a previsão de investimento de $80 \%$ do orçamento total. Apenas cinco

\begin{tabular}{|c|c|c|c|c|c|}
\hline & \multicolumn{5}{|c|}{$\begin{array}{c}\text { Previsão de Orçamento } \\
\text { (porcentagem do total 175,6) }\end{array}$} \\
\hline & $90 \%$ & $80 \%$ & $70 \%$ & $60 \%$ & $50 \%$ \\
\hline \multicolumn{6}{|c|}{ Receita gerada } \\
\hline & 83,68 & 76,58 & 68,39 & 61,84 & 51,63 \\
\hline \multicolumn{6}{|c|}{ Receita / custo } \\
\hline & 0,53 & 0,54 & 0,56 & 0,59 & 0,59 \\
\hline \multicolumn{6}{|c|}{ Número de canais não atendidos } \\
\hline Serviço 1 & 0 & 19,00 & 66,00 & 95,00 & 134,00 \\
\hline Serviço 2 & 12,11 & 38,11 & 55,56 & 73,78 & 105,33 \\
\hline \multicolumn{6}{|c|}{ BTSs não alocadas } \\
\hline & - & 8 & $3-8-9$ & $1-7-8-9$ & $\begin{array}{c}4-7-8 \\
9-10\end{array}$ \\
\hline \multicolumn{6}{|c|}{ Sistemas escolhidos (x E1) } \\
\hline HDSL & 0 & 0 & 0 & 0 & 0 \\
\hline $\begin{array}{l}\text { Óptico } \\
(1 / 2 / 4 / 8)\end{array}$ & $0 / 0 / 2 / 8$ & $0 / 0 / 3 / 10$ & $0 / 0 / 3 / 7$ & $0 / 0 / 3 / 7$ & $0 / 0 / 3 / 7$ \\
\hline $\begin{array}{l}\text { Rádio } \\
(2 / 4 / 8)\end{array}$ & $0 / 5 / 0$ & $1 / 1 / 0$ & $0 / 2 / 0$ & $0 / 1 / 0$ & $0 / 0 / 0$ \\
\hline
\end{tabular}

Tabela 5: Soluções para o cenário do item 4.3.2.

BTSs $(4,5,7,8$ e 9) tiveram demanda reprimida. Pode-se observar também que, na busca de se obter a receita máxima, a BTS 8 nem teve quipamentos novos alocados.

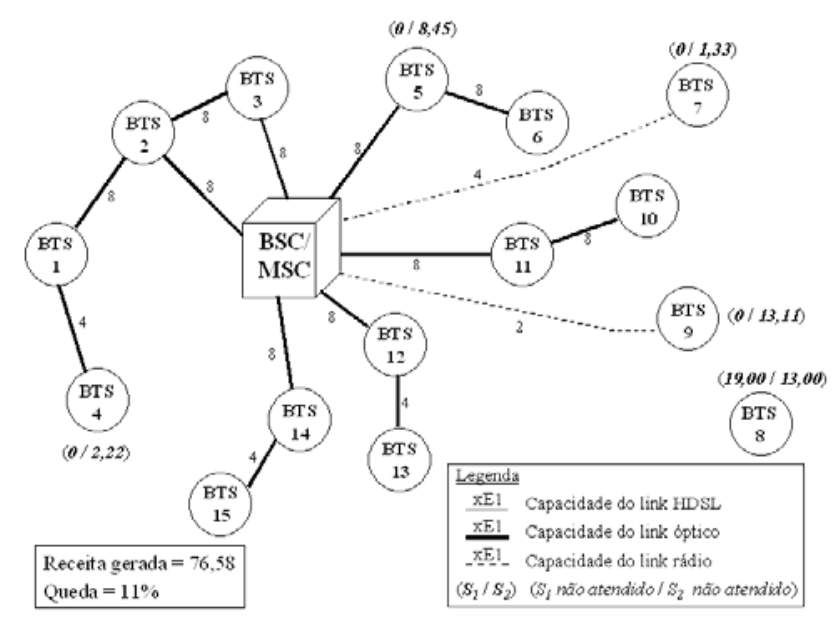

Figura 11: Configuração da rede para o cenário do item 4.3.2. 


\subsection{Aplicação do Modelo Fuzzy: Impacto da Demanda Imprecisa}

Durante as fases iniciais de operação de um sistema de acesso, a demanda em uma determinada área de atendimento tende a ser baixa. Quando os usuários da área tornam-se mais familiarizados com os serviços, os níveis de demanda tendem a aumentar gradualmente. Portanto o objetivo desta análise foi determinar o impacto sofrido pelo sistema (custos, receitas, etc) devido à imprecisão nos dados de demanda dos serviços.

Foram mantidas as mesmas condições de rede implantada, tecnologias candidatas e exigência de atendimento mínimo utilizadas no item 4.3. Números fuzzy triangulares (tabela 3) indicam os níveis de demanda (imprecisa) em cada nó de BTS. É assumido que o nível de confiança nos dados $(\alpha)$ é o mesmo em todos os nós de BTS.

Diante dos valores apresentados para a demanda dos serviços, três situações, em particular, são muito importantes. Primeiro, se o valor da demanda é o menor $\left(\underline{\mathbf{d}}_{s i}\right)$ para todos os serviços, uma condição que não está inclusa no equivalente de Adamo, a solução corresponde à configuração "mínima" de rede. Segundo, usando $\mathbf{D}_{s i}(\alpha=1)$, obtém-se a melhor configuração de rede para os valores de demanda mais confiáveis. Finalmente, se for utilizado o valor de demanda superior $\left(\bar{d}_{s i}\right.$, ou $\left.\alpha=0\right)$, é possível obter a configuração de rede que atende a situação mais otimista do ponto de vista de receita. A partir destes resultados o planejador passa a conhecer os limitantes de custo e receita de seu sistema.

As outras possibilidades de ocorrência a serem analisadas através do equivalente de Adamo para os números fuzzy geram soluções que se enquadram num meio-termo entre os limites obtidos para os valor de maior pertinência $(\alpha=1)$ e o valor máximo $(\alpha=0)$ da demanda.

Para ambos os cenários de controle de demanda, por nó de acesso ou por serviço, foi considerado o mesmo procedimento de resolução:

1 - Encontrou-se a configuração de rede, pelo critério de minimização de custo, capaz de atender toda a demanda prevista, resultante de cada grau de confiança considerado;

2 - Para cada solução obtida anteriormente, foi imposta uma queda de $15 \%$ no orçamento. A rede foi novamente dimensionada, objetivando a receita máxima.

\subsubsection{Análise Técnico-Econômica pelo Controle de Demanda por Nó de Acesso}

A tabela 6 lista os índices econômicos e as configurações de atendimento para as principais situações de demanda pre-

\begin{tabular}{|c|c|c|c|}
\hline \multirow{2}{*}{$\begin{array}{l}\text { Solução } \\
\text { min-custo }\end{array}$} & \multicolumn{3}{|c|}{ Demanda prevista } \\
\hline & $\underline{\mathbf{d}}_{s i}$ & $\mathbf{D}_{s i}$ & $\bar{d}_{s i}$ \\
\hline \multicolumn{4}{|l|}{ Custo } \\
\hline & 130,10 & 156,20 & 175,60 \\
\hline \multicolumn{4}{|l|}{ Receita gerada } \\
\hline & 37,50 & 61,80 & 86,10 \\
\hline \multirow{2}{*}{$\begin{array}{l}\text { Solução } \\
\text { max-receita }\end{array}$} & \multicolumn{3}{|c|}{ (com corte de $15 \%$ no orçamento) } \\
\hline & & & \\
\hline \multicolumn{4}{|l|}{ Receita gerada } \\
\hline & - & 57,42 & 77,24 \\
\hline \multicolumn{4}{|l|}{ Receita / custo } \\
\hline & - & 0,43 & 0,52 \\
\hline \multicolumn{4}{|c|}{ Número de usuários não atendidos } \\
\hline Serviço 1 & - & 6,00 & 21,50 \\
\hline Serviço 2 & - & 18,89 & 33,56 \\
\hline \multicolumn{4}{|l|}{ Rede alocada } \\
\hline HDSL & - & 0 & 0 \\
\hline Óptico(1/2/4/8) & - & $0 / 0 / 4 / 8$ & $0 / 0 / 5 / 5$ \\
\hline Rádio (2/4/8) & - & $3 / 0 / 0$ & $3 / 2 / 0$ \\
\hline
\end{tabular}

Tabela 6: Principais resultados do item 4.4.1.

vista, admitindo-se o controle de demanda por nó de acesso.

A tecnologia utilizando modems ópticos é predominante. Mas deve-se ressaltar que a rede já existente é fator importante na escolha das tecnologias.

Pode-se observar também que para a condição de demanda mínima, um corte de $15 \%$ no orçamento proibiu a obtenção de uma solução de rede factível.

É interessante notar que a abordagem de maximização de receita torna-se mais eficiente (veja a relação receita/custo) para as situações com previsão de demanda mais elevada. Nestas condições, a hierarquia dos equipamentos alocados tende a ser maior, o que permite um melhor compartilhamento baseado no ganho de escala.

As receitas obtidas para alguns valores intermediários de demanda (em função dos níveis de confiança) são apresentadas na figura 12. Para facilitar a análise são indicados também os valores de receita para a condição de atendimento total. Em geral, quando a confiança sobre os valores de demanda diminui $(\alpha \rightarrow 0)$, a receita aumenta. $\mathrm{O}$ atendimento dos serviços é, obviamente, penalizado quando o orçamento é reduzido. O corte de $15 \%$ no orçamento causa, em média, um corte de $11 \%$ na receita. 


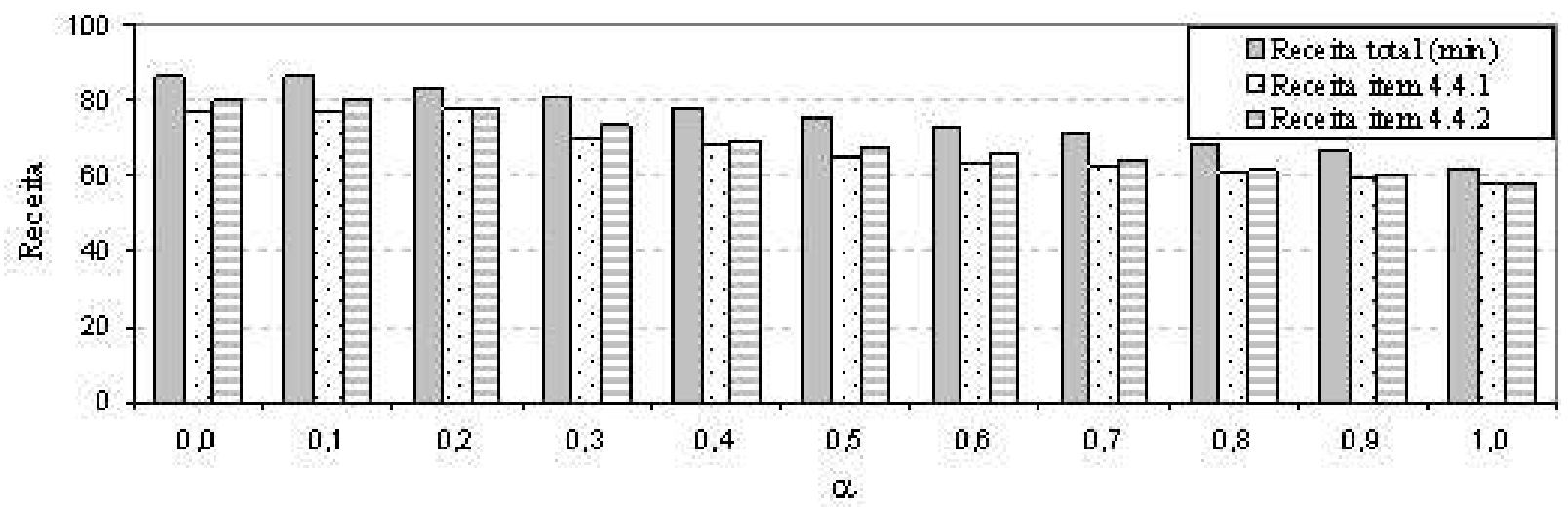

Figura 12: Receita x Nível de confiança (cenários 4.4.1 e 4.4.2).

\subsubsection{Análise Técnico-Econômica pelo Controle de Demanda por Serviço}

A tabela 7 lista os índices econômicos e as configurações de atendimento para as principais situações de demanda prevista, admitindo-se o controle de demanda por serviço. Para facilitar a análise, os valores obtidos da solução de mínimo custo também são apresentados na tabela 7 .

A tecnologia óptica continua sendo a mais apropriada; porém, a rede é dimensionada de forma a compartilhar melhor os meios de transmissão, principalmente para a condição de demanda máxima, onde se tem uma grande utilização de equipamentos com maior hierarquia.

Diferentemente do ocorrido no item 4.4.1, aqui o corte de $15 \%$ no orçamento não impossibilitou a obtenção de uma solução factível para a previsão de demanda mínima $\left(\underline{\mathbf{d}}_{s i}\right)$. A solução foi garantida através de uma rede "enxuta", com os equipamentos alocados em baixa hierarquia, e sacrificando a demanda total das BTS 8 e 14 .

Embora também não esteja explicito na tabela 7, a demanda reprimida para a condição de demanda máxima $\left(\bar{d}_{s i}\right)$ é basicamente toda aquela que estava prevista para a BTS 8 .

As receitas obtidas para alguns valores intermédiarios de demanda também são apresentados na figura 12. Pode-se observar que devido à possibilidade de atender a demanda sem se preocupar com o atendimento mínimo por região da rede, a receita obtida aqui é ligeiramente maior (2\%, em média) do que aquela apresentada no item 4.4.1.

\section{CONCLUSÕES}

A perspectiva de um ambiente de competição está levando as empresas de telecomunicações a assumirem uma nova pos-

\begin{tabular}{|c|c|c|c|}
\hline \multirow{2}{*}{$\begin{array}{l}\text { Solução } \\
\text { min-custo }\end{array}$} & \multicolumn{3}{|c|}{ Demanda prevista } \\
\hline & $\underline{\mathbf{d}_{s i}}$ & $\mathbf{D}_{s i}$ & $\bar{d}_{s i}$ \\
\hline \multicolumn{4}{|l|}{ Custo } \\
\hline & 130,10 & 156,20 & 175,60 \\
\hline \multicolumn{4}{|l|}{ Receita gerada } \\
\hline & 37,50 & 61,80 & 86,10 \\
\hline Solução & \multicolumn{3}{|c|}{ (com corte de $15 \%$ no orçamento) } \\
\hline max-receita & & & \\
\hline \multicolumn{4}{|l|}{ Receita gerada } \\
\hline & 33,94 & 57,49 & 79,96 \\
\hline \multicolumn{4}{|l|}{ Receita / custo } \\
\hline & 0,31 & 0,43 & 0,54 \\
\hline \multicolumn{4}{|c|}{ Número de usuários não atendidos } \\
\hline Serviço 1 & 16,00 & 0,00 & 19,00 \\
\hline Serviço 2 & 9,78 & 21,56 & 21,22 \\
\hline \multicolumn{4}{|l|}{ Rede alocada } \\
\hline HDSL & 0 & 0 & 0 \\
\hline Óptico(1/2/4/8) & $0 / 2 / 3 / 1$ & $0 / 0 / 4 / 8$ & $0 / 0 / 4 / 9$ \\
\hline Rádio (2/4/8) & $6 / 1 / 0$ & $3 / 0 / 0$ & $0 / 1 / 0$ \\
\hline
\end{tabular}

Tabela 7: Principais resultados do item 4.4.2.

tura quanto ao planejamento de suas redes. A velocidade com que surgem novas tecnologias e demandas por novos serviços obriga as empresas a tomarem decisões de forma cada vez mais rápida. A rede de acesso, componente do sistema de telecomunicações, por representar o segmento responsável pelo atendimento individual de cada usuário, é o foco imediato destas transformações.

Dúvidas sobre quais serviços oferecer, e sobre as suas ren- 
tabilidades, são inevitáveis. Portanto, faz-se necessário um planejamento da rede, enfatizando a receita gerada pelos serviços oferecidos, além do custo envolvido, em um ambiente capaz de analisar várias opções tecnológicas.

Neste trabalho, é apresentado um modelo bastante flexível que pode ser aplicado no planejamento de sistemas de acesso multi-serviço, fixo e/ou móvel, em situações nas quais existe imprecisão sobre os valores adotados como demanda dos serviços. O dimensionamento dos componentes da rede é a atividade mais explorada. A rede é vista como um grafo e a modelagem de maximização de receita é traduzida como um problema de programação linear inteira mista, o qual obedece restrições técnicas de capacidade e orçamento. A imprecisão é abordada de acordo com o conceito de números fuzzy.

Em relação aos cenários analisados, é interessante ressaltar, do ponto de vista técnico-econômico, as seguintes constatações sobre os resultados obtidos:

- a infra-estrutura da rede instalada é um fator decisivo para a dimensionamento do sistema. Pôde-se observar que, para se obter uma maior receita, as soluções utilizando modems ópticos dominaram o planejamento em situações de baixa disponibilidade de cabos metálicos;

- à medida que a hierarqua dos equipamentos alocados aumenta, consequência direta de cenários mais otimistas para a demanda, o compartilhamento de recursos torna-se mais acentuado, o que acaba melhorando o retorno (receita/investimento) financeiro do sistema;

- em termos de receita gerada, o controle de demanda por serviço apresentou-se mais atraente que o controle de demanda por nó de acesso. Isto evidencia a necessidade de investimento adicional quando se tem o interesse de se manter uma mesma receita, porém, com obrigações de atendimento em setores especificos da rede.

Considerando o ponto de vista da modelagem, cuja finalidade é auxiliar o planejador ao longo do processo de decisão, verifica-se que a metodologia proposta é capaz de refletir todas as principais situações encontráveis no ambiente das telecomunicações. A flexibilidade quanto a variações nos cenários possíveis de serem contemplados é uma das suas principais virtudes. Podem-se destacar:

- capacidade de reconhecer uma rede existente. Esta é uma característica imprescindível para um eventual planejamento estagiado, onde o dimensionamento dos equipamentos em uma etapa é fortemente dependente da rede dimensionada no estágio anterior;
- inclusão de tecnologias de rede que otimizam a utilização da rede metálica para o provimento de serviços faixa-larga;

- previsão de competição entre as tecnologias (e topologias) mais viáveis para os sistemas de acesso;

- possibilidade de realizar um planejamento multiserviço;

- possibilidade de atribuir prioridades no atendimento dos serviços;

- alta interatividade. Devido à modelagem da imprecisão, situações de análise de sensibilidade podem ser facilmente geradas pelo planejador;

- possibilidade de um planejamento que permite, ao mesmo tempo, obedecer à restriçoes de contrato (como por exemplo, o atendimento mínimo de determinado serviço, em uma região em particular da rede) e "visualizar"os cenários mais lucrativos (onde investir e como investir) dentro das suas condições financeiras de investimento.

Como extensões deste trabalho pode-se destacar:

- o desenvolvimento de um algoritmo para escolha mais criteriosa dos valores pontuais do nível de confiança $(\alpha)$. O objetivo é otimizar a quantidade de problemas PLIM a serem resolvidos, melhorando o desempenho computacional;

- propostas de resolução para os problemas PLIM baseadas em algoritmos genéticos. O objetivo, novamente, é melhorar o desempenho computacional, ao sugerir a obtenção de uma solução de compromisso de boa qualidade;

- adaptações no sentido de incorporar novas tecnologias e serviços;

- a adaptação do modelo para contemplar a dependência existente entre a demanda do serviço e a sua receita unitária;

- na mesma linha de pesquisa direcionada à receita, uma outra possibilidade de interesse é o planejamento orientado ao lucro. Esta abordagem é consideravelmente mais complicada do que a apresentada neste trabalho, pois implica em avaliar a evolução do sistema ao longo do tempo. 


\section{REFERÊNCIAS}

Asga (2005). Especificações Técnicas de Modems Ópticos. Website do Fabricante AsGa: http://www.asga.com.br, Janeiro.

Balakrishnan, A., T.L. Magnanti, A. Shulman and R.T. Wong (1991). Models for Planning Capacity Expansion in Local Access Telecommunication Networks. Annals of Operations Research, n. 33, pp. 239-284.

Bazaraa, M.S., J.J. Jarvis and H.D. Sherali (1990). Linear Programming and Network Flows. $2^{\text {nd }}$ ed., Willey, New York.

Campos, L. and J. L. Verdegay (1989). Linear Programming Problems and Ranking of Fuzzy Numbers. Fuzzy Sets and Systems: 32, pp. 1-11.

Carlson, C.M.F and G. Authié (2001). Optimized Design of LMDS Cells: Site Location, Equipment Sizing and Users Homing. Procs. of the Third IEEE International Conference on Mobile and Wireless Communication Networks - MWCN'2001, Recife, Brasil, pp. 111118.

CPLEX Optimization. Inc. (1994). Using the CPLEX Callable Library.

Crema, A. (2002). The Multiparametric 0-1 Integer Linear Programming Problem: A Unified Approach. European Journal of Operational Research, n.139, pp. 511520.

DeSousa, M.A., J.T. Machado, R.V. Ribeiro e C.M.F Carlson (2003). Uma proposta de Modelagem TécnicoEconômica para o Planejamento Estratégico de Sistemas de Acesso. Anais do XX Simpósio Brasileiro de Telecomunicações - SBT'03, Rio de Janeiro-RJ, pp. 834839.

DeSousa, M.A., J.T. Machado, R.V. Ribeiro e C.M.F. Carlson (2003a). A Revenue-Focused Planning Model of Cellular Networks with Imprecise Demand Constraints. Procs. of the 7th World Multiconference on Systemics, Cybernetics and Informatics - SCI 2003, Orlando USA, Vol. IX, pp 320-325.

DeSousa, M.A. et alii (2001). Cellular Networks Infrastructure: An Optimized Planning. Procs. of the 9th International Conference on Telecommunications Systems Modelling and Analysis, Dallas -USA, pp. 331-340.

DeSousa, M.A., R. V. Ribeiro, J.T. Machado, C.M.F. Carlson and J.R.F. Formigoni (2001a). Income-oriented Broadband Access Planning with xDSL and APON-FTTC. Proceedings of the Third International Workshop on Design of Reliable Communication Networks (DRCN2001), Budapest, pp. 330-337.

DeSousa, M.A., J.T. Machado, R.V. Ribeiro and C.M.F. Carlson (2001b). Risk Evaluation in Broadband Telecommunications Planning Under Services' Imprecise Penetration - A Fuzzy Approach. Procs. of the FUZZ-IEEE 2001, Melbourne-AU, vol. II, p\#136.

DeSousa, M.A. e C.M.F. Carlson (2000). Avaliação do Risco Técnico-Econômico no Planejamento da Rede de Acesso Multi-serviço - Uma Abordagem Fuzzy. Anais do XIII Congresso Brasileiro de Automática-CBA2000, Florianópolis-SC, pp. 2192-2197.

DeSousa, M.A., C.M.F. Carlson, J.R.F. Formigoni e R.V. Ribeiro (1999). Planejamento da Rede de Acesso Orientado à Maximização de Receitas. Anais do XVII Simpósio Brasileiro de Telecomunicações - SBT999, Vila Velha-ES, pp. 389-394.

Ericsson (2005). Especificações Técnicas de Sistemas de Rádio Microondas. Website do Fabricante Ericsson: http://www.ericsson.com/products, Janeiro.

Gavish, B. and S. Sridhar (1995). Economic Aspects of Configuring Cellular Networks. Wireless Networks 1, pp. $115-128$

Hummel, P.R.V. e M.R.B. Taschner (1992). Análise e Decisão sobre Investimentos e Financiamentos. Atlas, $3^{a}$. ed., São Paulo.

Humphrey, M. and J. Freeman (1997). How xDSL Supports Broadband Services to the Home. IEEE Network (Jan./Feb.), pp. 14-23.

Ims, L.A., B.T. Olsen, D. Myhre and K. Stordahl (1998). Examining the Technologies, Migration Strategies and Economics of Broadband Wireline Access Network Architectures. Procs. of the IIR Conference, London-UK, pp. 1-10.

Ims, L.A., K. Stordahl and B.T. Olsen (1997). Risk Analysis of Residential Broadband Upgrade in a Competitive and Changing Market. IEEE Communications Magazine (Feb.), pp. 96-103.

Kubat, P. and J.M. Smith (2001). A Multi-period Network Design Problem for Cellular Tececommunication Systems. European Journal of Operacional Research, n. 134, pp. 439-456.

Machado, J.T., M.A. DeSousa, C.M.F. Carlson e R.V. Ribeiro (2003). A Genetic Approach to Equipment Allocation and Topology Choice in Wireless MultiService Networks. Procs. of the 9th International Conference on Computer, Communication and Control 
Technologies-CCCT2003, Orlando -USA, vol. II, pp. 404-409.

Martinelli, C.E. e F.R. Carneiro (1996). Evolução das Redes Híbridas Fibra/Coaxial. Anais do XII Seminário de Redes do Sistema Telebrás, Brasília, pp. 247-258.

Moura, A.F. e A.S. Garcia (2000). Uma Visão de Redes Multicamadas com Enfoque em Mapeamento de Redes ATM sobre Redes SDH. Anais do XIII Congresso Brasileiro de Automática - CBA2000, Florianópolis-SC, pp. 2198-2203.

Olsen, B.T. et alii. (1996). Techno-Economic Evaluation of Narrowband and Broadband Access Network Alternatives and Evolution Scenario Assessment. IEEE Journal on Selected Areas in Communications, vol. 14, n. 6, pp. 1184-1204.

Pedrycz, W. and F. Gomide (1998). An Introduction to Fuzzy Sets: Analysis and Design. MIT Press, Cambridge, MA.

Sahinidis, N.V. (2004). Optimization under Uncertainty: State-of-the-art and Opportunities. Computer \& Chemical Engineering 28 (6-7), pp. 971-983.

Sexton, M. and A. Reid (1997). Broadband Networking: ATM, SDH and Sonet. Artech House, Boston.

Simens (2005). Especificações Técnicas de Sistemas de Rádio Microondas. Website do Fabricante Siemens: http://www.siemens.com, Janeiro.

Toledo, A.P. (2001). Redes de Acesso em Telecomunicações. Makron Books, São Paulo.

Ufongene, C.M. (1999). A Model for the Cost Analysis of Wireless Access Architectures. Bell Labs Technical Journal, July-September, pp. 134-154.

Yacoub, M.D. (2001). Wireless Technology: Protocols, Standards and Techniques. CRC Press, London.

Zhou, C., D. Qian, N. Pissinou and K. Makki (2003). Pricing and Revenue Maximization for Wireless CDMA Networks. Procs. of the 7th World Multiconference on Systemics, Cybernetics and Informatics - SCI 2003, Orlando - USA. 